\title{
Intrinsic and selective activity of functionalized carbon nanotube/ nanocellulose platforms against colon cancer cells
}

\author{
J.M. González-Domínguez ${ }^{\text {a, * }}$, L. Grasa ${ }^{\text {b,c, d,*** }}$, J. Frontiñán-Rubio ${ }^{\mathrm{e}}$, E. Abás ${ }^{\mathrm{b}}$, \\ A. Domínguez-Alfaro ${ }^{\mathrm{f}}$, J.E. Mesonero ${ }^{\mathrm{b}, \mathrm{c}, \mathrm{d}}$, A. Criado ${ }^{\mathrm{f}, \mathrm{g}}, \mathrm{A}$. Ansón-Casaos ${ }^{\mathrm{a}}$ \\ ${ }^{a}$ Instituto de Carboquímica, ICB-CSIC, Miguel Luesma Castán 4, 50018 Zaragoza, Spain \\ b Departamento de Farmacología, Fisiología y Medicina Legal y Forense, Facultad de Veterinaria, Universidad de Zaragoza, Miguel Servet, 177, Zaragoza 50013, Spain \\ ${ }^{\mathrm{c}}$ Instituto de Investigación Sanitaria de Aragón (IIS-Aragón), Zaragoza, Spain \\ d Instituto Agroalimentario de Aragón - IA2 -, Universidad de Zaragoza - CITA, Zaragoza, Spain \\ ${ }^{\mathrm{e}}$ Department of Medical Sciences, Ciudad Real Medical School, University of Castilla-La Mancha, Ciudad Real, Spain \\ ${ }^{\mathrm{f}}$ Center for Cooperative Research in Biomaterials (CIC biomaGUNE), Basque Research and Technology Alliance (BRTA), 20014 Donostia-San Sebastián, Spain \\ ${ }^{g}$ Centro de Investigacións Científcas Avanzadas (CICA), Universidade da Coruña, A Coruña, Spain
}

\section{A R T I C L E I N F O}

\section{Keywords:}

Carbon nanotubes

Nanocellulose

Fluorescein

Caco-2 cells

Chemotherapy

Theranostics

\begin{abstract}
A B S T R A C T
Given their large surface area and versatile chemical reactivity, single-walled carbon nanotubes (SWCNTs) are regarded as the basis of new pharmacological complexes. In this study, SWCNTs are chemically functionalized with fluorescein, folic acid, and capecitabine, a drug that is commonly used against colorectal cancer. These functionalized SWCNTs are dispersed in water by taking advantage of their synergistic interaction with type-II nanocrystalline cellulose (II-NCC), and the resulting colloidal system is tested in vitro on both normal (differentiated) and cancerous (proliferative) human colon cells (Caco-2). The functionalized SWCNT/II-NCC hybrids show a higher activity than the reference (capecitabine) against the Caco-2 cancer cell line. However, this effect appears to be intrinsically associated with the SWCNT/II-NCC complex, particularly boosted by fluorescein, as the presence of capecitabine is not required. In addition, confocal microscopy fluorescence imaging using cell cultures highlights the enormous potential of this nanohybrid platform for colon cancer theranostics.
\end{abstract}

\section{Introduction}

The potential applications of nanoparticulate materials include cancer diagnosis and treatment [1]. Current research in this field aims to combine multimodal imaging and therapeutic activity in a single functional complex [2], which is the conceptual basis of nanoscale theranostics. Imaging modes are typically based on fluorescent centers [3-8], nuclear magnetic resonance contrast agents [9-11], photoacoustic detection $[11,12]$, tomography with $\gamma$-radiation [13], and so on. With regard to treatment methods, nanoparticles enable photothermal therapies $[6,14]$ and can be loaded with radiotherapy and chemotherapy agents $[3,9,13,15]$. In addition, the drug efficiency can be improved by implementing positive targeting strategies [1], such as the well-known receptor-mediated targeting by the folate chemical group $[3,4,6,16]$. Most specific novel methods are oriented to cellular organelles [17-19], and can be guided magnetically [20], or accommodate controlled catalytic release [21].

Carbon nanotubes (CNTs) offer great benefits as drug carriers as they can act as "nanoneedles" for internalization and exhibit a large surface area (external and internal) and versatile surface chemistry for functionalization with multiple active centers $[3,13,22]$. In addition, toxicity drawbacks due to their aspect ratio and metal impurities can be avoided by applying suitable cutting, purification, and functionalization strategies [20]. Their hydrophobic character, which may make them incompatible with biological media, can be tuned by chemical functionalization and colloidal stabilization [9]. In principle, stabilization via physical interactions avoids disruption of the chemical structure and optoelectronic properties of the nanostructure. However, particular attention must be paid to the surfactant toxicity at the required concentration [23,24]. In this context, novel biopolymer-based

\footnotetext{
* Corresponding author.

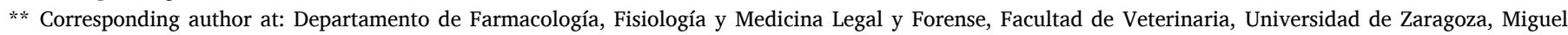
Servet, 177, Zaragoza 50013, Spain.

E-mail addresses: jmgonzalez@icb.csic.es (J.M. González-Domínguez), lgralo@unizar.es (L. Grasa).
} 


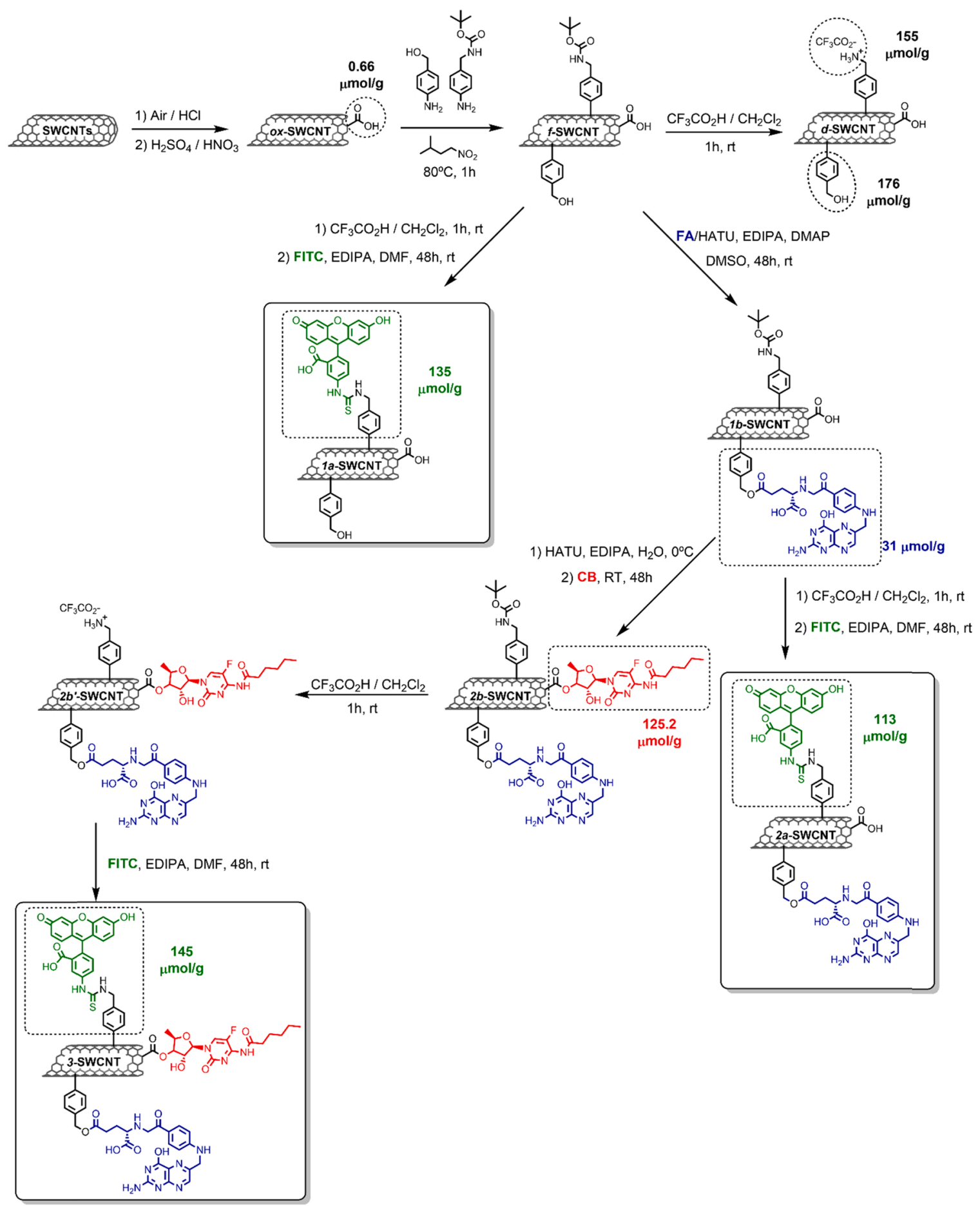

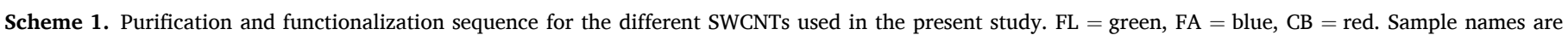
shown on the nanotubes.

nanostructures, such as nanocellulose, are emerging as new paradigms for the efficient stabilization of CNTs in water within a biocompatible and environmentally friendly framework [25]. Nanocellulose is a crystalline fibrous nanostructure that is typically derived from plant sources, is biocompatible and can be prepared by way of environmentally friendly processes. Nanocellulose interacts with CNTs via physical interactions to form hybrid nanostructures that become highly stable when dispersed in water $[26,27]$. In this synergistic manner, nanocellulose exhibits excellent properties as a stabilizer for CNTs compared to inorganic surfactants, biomolecules, and other classical polysaccharides.

It has been shown previously that some of the most common drugs against colon cancer can be carried by nanoparticulate vehicles $[28,29]$. For example, fluorouracil has been loaded onto the external surface of CNTs by chemical functionalization reactions [20] or physical adsorption [30]. Another drug, namely irinotecan, has been encapsulated within CNTs [31] and physically adsorbed on CNT surfaces [32]. Similarly, capecitabine, which is the drug of choice in the present study, has been successfully entrapped in polymeric nanoparticles [33].

The objective of this research was to synthesize a water-dispersible 
pharmacological platform based on single-walled carbon nanotubes (SWCNTs) and nanocellulose. A fluorophore (fluorescein) and a targeting molecule (folic acid) were covalently linked to the SWCNTs to facilitate detection and targeting, respectively, of cancer cells. The anticancer drug capecitabine was also chemically grafted to the SWCNT surface and the resulting pharmacological complex was administered to human colon (Caco-2) cells cultured to exhibit two different morphologies: cancer (proliferative) and normal (enterocyte-like differentiation). It was observed that the antiproliferative effect of the synthesized complex is superior to that of capecitabine alone (reference substance). Surprisingly, it was discovered that the capecitabine group is not needed on the complex to achieve the anticancer effect, and several control experiments were performed to identify the active principle on the SWCNT platform. It is concluded that the SWCNT/nanocellulose hybrid itself exerts a potent anticancer effect. It is also shown that the chemotherapeutic activity is compatible with the linkage of imaging tags such as a fluorophore. As such, this research describes for the first time the high anticancer activity and diagnostic possibilities of a SWCNT/nanocellulose hybrid.

\section{Experimental}

\subsection{Materials}

SWCNTs (AP-SWNT grade) were purchased from Carbon Solutions Inc. (Riverside, CA, USA). Sigma-Aldrich was the provider for all solvents and acids, together with microcrystalline cellulose (MCC) powder, $20 \mu \mathrm{m}$, (\#310697), fluorescein isothiocyanate isomer I $(\geq 90 \%$, \#F7250), capecitabine ( $\geq 99.0 \%$, \#00921), folic acid ( $\geq 97 \%$, \#F7876), 4-[( $N$-Boc)aminomethyl]aniline (97\%, \#525626), 4-aminobenzyl alcohol (98\%, \#191558), isopentyl nitrite (97\%, \#59160), $N, N$-diisopropylethylamine (EDIPA, \#D125806), trifluoroacetic acid (99\%, \#T6508), human albumin (ALB, $\geq 96 \%$, \#A9731), molecular biologygrade dimethyl sulfoxide (DMSO, \#D8418), the benzotriazol derivative HATU (97\%, \#445460), 4-(dimethylamino)pyridine (DMAP, 98\%, \#39405), Kaiser test kit (\#60017), L-ascorbic acid (\#A92902), Lglutathione reduced (\#G4251), all of which were used as received. Poly (ethylene glycol) (PEG, $\mathrm{M}_{\mathrm{w}} \sim 10,000$, Fluka BioChemika, \#92897), gellan gum (GG, Fluka), and sodium deoxycholate (DOC, 99\%, Acros Organics) were alsoused as received. Phosphate buffer solution (PBS) $1 \mathrm{x}$ was prepared in-house to the standard recipe but with less $\mathrm{NaCl}(20 \mathrm{mM})$ than the commercial version $(137 \mathrm{mM})$.

\subsection{Chemical functionalization of SWCNTs}

Pristine SWCNT powders were purified by thermal treatment in air and reflux in $\mathrm{HCl}$. The SWCNTs were then functionalized with carboxylic acid groups by refluxing in $\mathrm{H}_{2} \mathrm{SO}_{4}: \mathrm{HNO}_{3}$. Details of the preparation and characterization of these $o x$-SWCNTs have been published elsewhere [34].

The subsequent covalent functionalization roadmap is displayed in Scheme 1. A primary functionalization stage with $o x$-SWCNTs was carried out via a one-pot double diazonium-based arylation reaction inspired by a previous work [35]. A sequential routine was then followed to covalently anchor fluorescein (FL) to the benzylamine moieties, folic acid (FA) to the benzyl alcohol moieties, and capecitabine (CB) to the carboxylic acid groups. The details of each step are described in the Supplementary Materials.

\subsection{Preparation of nanocellulose}

As discussed in the introduction, the synthesis of NCC was carried out according to a methodology developed in our laboratory [36]. In a typical experiment, $10 \mathrm{~g}$ of commercial MCC was soaked in $45 \mathrm{~mL}$ of ultrapure water in a round-bottomed flask, then dispersed with the aid of an ultrasonic bath for $10 \mathrm{~min}$. The mixture was then cooled to $0{ }^{\circ} \mathrm{C}$ and $45 \mathrm{~mL}$ of $98 \%$ sulfuric acid was added dropwise, under constant magnetic stirring. Immediately after addition of the last droplet of acid, the flask was removed from the ice bath and transferred to a heating plate. At this point, the procedure varied according to the type of NCC to be obtained. For NCC type I, the reaction medium was heated at $70{ }^{\circ} \mathrm{C}$ for $10 \mathrm{~min}$, while for type II it was heated at $27^{\circ} \mathrm{C}$ for $5 \mathrm{~h}$. Once the heating step had finished, the reaction medium was poured onto an approximately 10 -fold excess of cold deionized water in a $1 \mathrm{~L}$ beaker and left overnight at $4{ }^{\circ} \mathrm{C}$, in order to favor sedimentation. After decanting, the supernatant liquid was neutralized by dialysis. To that end, the aqueous dispersion was poured into a dialysis membrane (SpectraPor ${ }^{\circledR}$, Spectrum Labs, regenerated cellulose, 6-8 kDa cutoff molecular weight) immersed in $5 \mathrm{~L}$ of ultrapure water. The dialysis water was changed periodically until neutral $\mathrm{pH}$ was achieved in the washing waters. The dialyzed medium was then centrifuged at around $9300 \mathrm{rcf}$, the supernatant liquid was isolated and subsequently freeze-dried in order to use the NCC as a fine and light powder. Full characterization of the NCC can be found in a previous report [36].

\subsection{Preparation of SWCNT colloids with aqueous dispersants}

Initial stability studies were carried out by dispersing $o x$-SWCNT (at $1 \mathrm{mg} / \mathrm{mL}$ ) with different dispersants, previously dissolved or suspended in water, at a concentration of 1 (MCC and types I and II NCC), 3 (GG), 5 (DOC) or $10 \mathrm{mg} / \mathrm{mL}$ (ALB, PEG). The procedure consisted of mixing both components and applying tip sonication (Hielscher UP400S at $24 \mathrm{kHz}, 0.5$ cycles, $60 \%$ amplitude) for $1 \mathrm{~h}$ while cooling externally with a water/ice bath. Subsequently, samples were centrifuged for $60 \mathrm{~min}$ in a Beckman Coulter L-100 XP ultracentrifuge equipped with a SW55Ti 3671 rotor and Beckman centrifugation tubes (ref. 326819). The centrifugation speed was $120,000 \mathrm{~g}$, except for DOC (200,000 g).

Due to their lower dispersibility in water compared to $o x$-SWCNTs, functionalized SWCNTs were mixed with the NCC/water dispersion and homogenized by cycles of ultrasound bath $(45 \mathrm{kHz})$ treatments for $30 \mathrm{~min}$ followed by long ( $\geq 24 \mathrm{~h}$ ) magnetic stirring periods, with no centrifugation. For biological tests, all SWCNT/NCC hybrid aqueous dispersions were prepared at $1 \mathrm{mg} / \mathrm{mL}$ SWCNTs and $5 \mathrm{mg} / \mathrm{mL}$ NCC.

\subsection{Characterization of SWCNT solids and SWCNT/nanocellulose colloids}

Thermogravimetric analysis (TGA) was performed using a TA Instruments Discovery system under air, starting from $100^{\circ} \mathrm{C}$ with a ramp of $10{ }^{\circ} \mathrm{C} / \mathrm{min}$ up to $600{ }^{\circ} \mathrm{C}$ after an isotherm at $100{ }^{\circ} \mathrm{C}$ for $20 \mathrm{~min}$. The covalently linked functional groups thermally desorbed by TGA were quantified by taking $500{ }^{\circ} \mathrm{C}$ as the reference temperature and applying the systematic described in ref [35]. X-ray photoelectron spectroscopy (XPS) measurements were performed using a SPECS Sage HR 100 spectrometer with a non-monochromatic aluminum X-ray source with a $\mathrm{K} \alpha$ line with an energy of $1486.6 \mathrm{eV}$ and $300 \mathrm{~W}$. The XPS data were fitted using CasaXPS software. Optical absorption spectroscopy of the dispersions was performed in $2 \mathrm{~mL}$ quartz cuvettes using Shimadzu UV-2401 PC and FTIR Vertex 70 Bruker spectrometers. Photoluminescence excitation and emission spectra were recorded using a Horiba Jobin Yvon Fluoromax-P. All spectra were recorded at room temperature using a $10 \mathrm{~mm}$ path-length quartz cuvette. Mass centrifugation yields were determined as the absorbance ratio at a wavelength of $850 \mathrm{~nm}$. Zeta $(\zeta)$ potential measurements were performed using a Z-Sizer Nano in U-shaped polymeric cells by Malvern. The Kaiser test was applied to quantify the amount of free primary amines present on the surface of the SWCNTs using a colorimetric method. The procedure described in reference [37] was applied. Transmission electron microscopy (TEM) was performed using a JEOL JEM-2100 F model EM-20014 equipped with a $200 \mathrm{kV}$ field emission electron gun. Samples were prepared by dilution in MilliQ water until $50 \mu \mathrm{g} / \mathrm{mL}$ and sonicated for 5-10 min. The dilution was then adjusted to $0.5 \mathrm{v} / \mathrm{v} \%$; no color was 

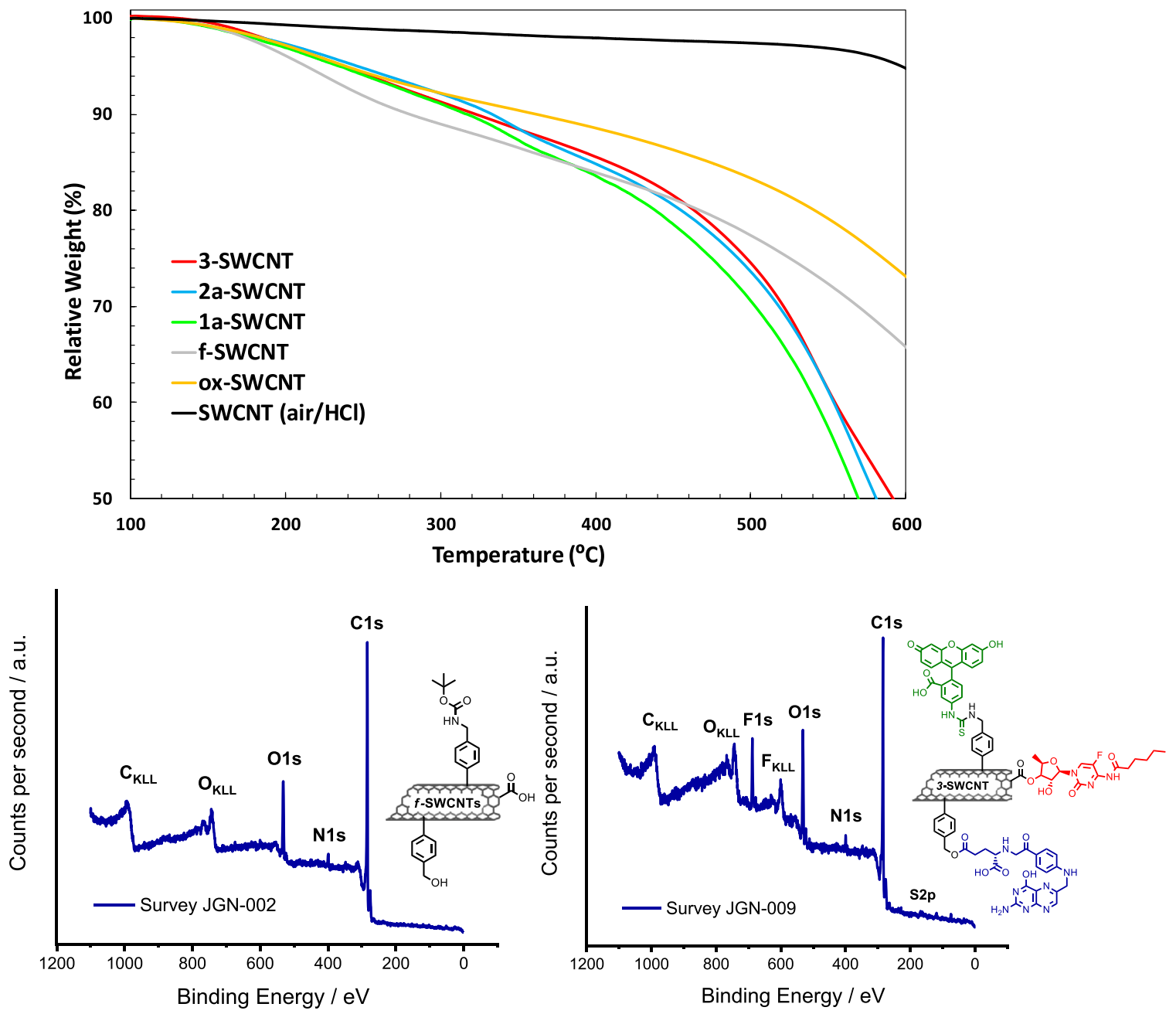

Fig. 1. Characterization of SWCNT functionalization by TGA (top panel). Survey XPS spectra for $f$-SWCNT (bottom left) and 3-SWCNT (bottom right).

Table 1

Atomic composition of two SWCNT samples obtained from the XPS survey spectra.

\begin{tabular}{lllll}
\hline Sample & C (at\%) & O (at\%) & N (at\%) & F (at\%) \\
\hline$f$-SWCNT & 85.4 & 2.4 & 12.2 & - \\
3-SWCNT & 81.3 & 2.3 & 11.4 & 5.0 \\
\hline
\end{tabular}

observed. Finally, a $5 \mu \mathrm{l}$ droplet of the sample was cast onto a copper grid coated with a carbon film and left to dry in air at room temperature for several hours.

\subsection{Cell culture}

This study was carried out using the human enterocyte-like cell line Caco-2/TC7 [38]. This cell line undergoes a process of spontaneous differentiation in culture that leads to the formation of a monolayer of cells expressing morphological and functional characteristics of the mature enterocytes. This differentiation process is growth-dependent, with the cells undergoing differentiation from undifferentiated proliferative crypt-type cells in the exponential growth phase to differentiated enterocyte-type cells in the stationary phase [39]. Caco-2/TC7 cells (passages 30-50) were cultured at $37{ }^{\circ} \mathrm{C}$ in an atmosphere of $5 \% \mathrm{CO}_{2}$ and maintained in high glucose DMEM supplemented with $2 \mathrm{mM}$ glutamine, $100 \mathrm{U} / \mathrm{mL}$ penicillin, $100 \mu \mathrm{g} / \mathrm{mL}$ streptomycin, $1 \%$ non-essential amino acids, and $20 \%$ heat-inactivated fetal bovine serum (FBS) (Life Technologies). For cell line maintenance, cells were passaged

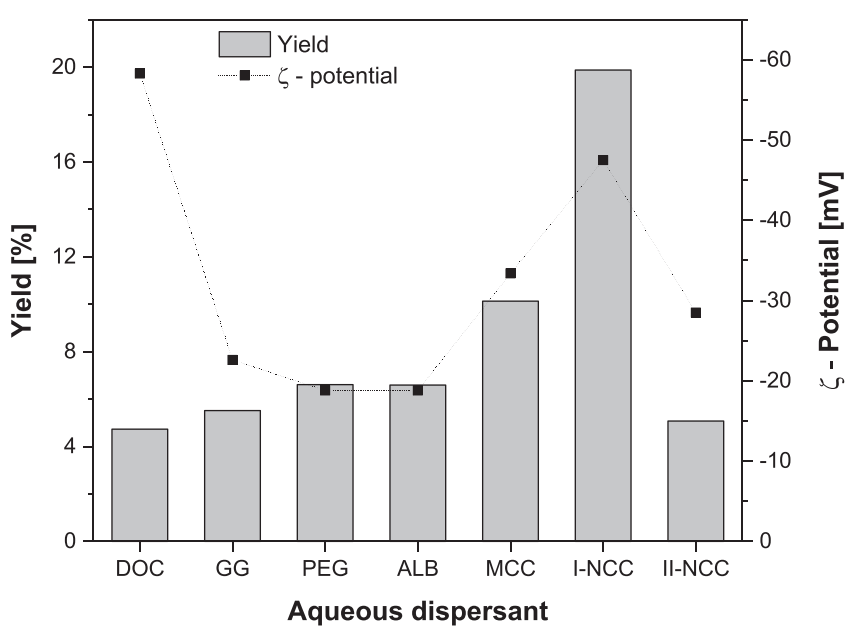

Fig. 2. Mass centrifugation yield and $\zeta$-potential of $o x$-SWCNT dispersions in different aqueous media. Spin velocity is $120,000 \mathrm{rcf}$ for $1 \mathrm{~h}$, except for DOC (200,000 rcf).

enzymatically with $0.25 \%$ trypsin- $1 \mathrm{mM}$ EDTA and subcultured on $25 \mathrm{~cm}^{2}$ plastic flasks at a density of $10^{4}$ cells $/ \mathrm{cm}^{2}$, replacing the culture medium every 2 days. With this culture density, the cells reach a cell confluence of $90 \%$ (where cell differentiation starts) at 7 days post-seeding, and complete cell differentiation is reached at 15 days 


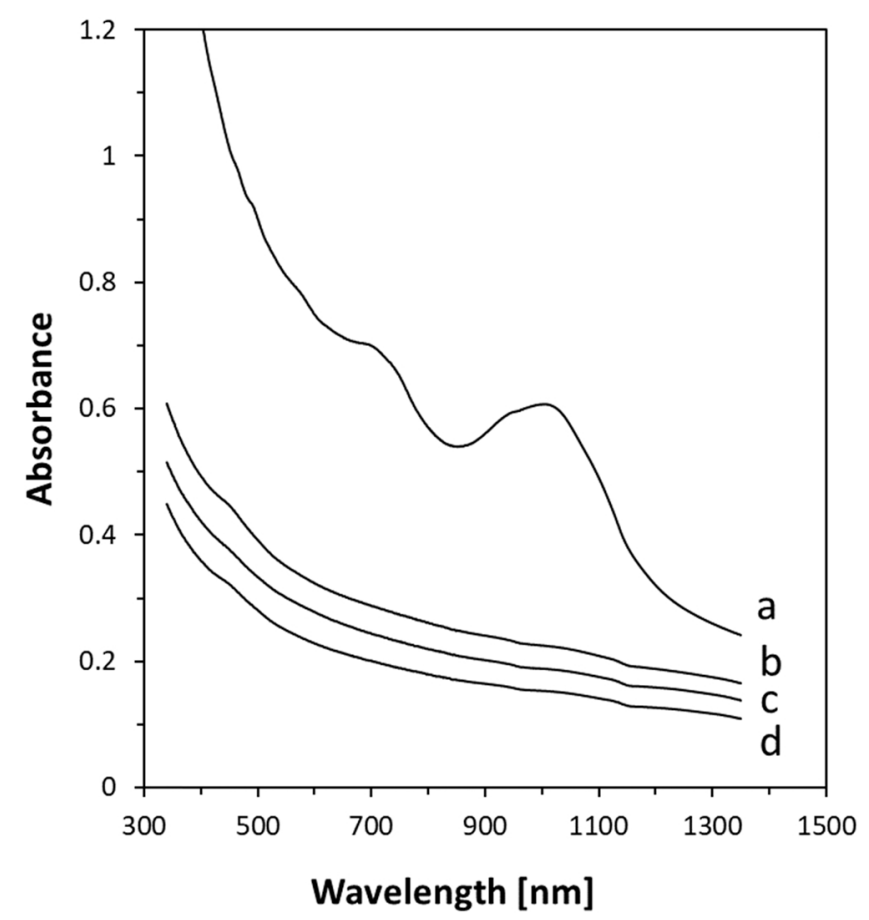

Fig. 3. Absorbance spectra of functionalized SWCNT hybrids with II-NCC: a) $o x$-SWCNT/II-NCC centrifuged at $120,000 \mathrm{~g}$ for $1 \mathrm{~h}$; b) $1 a$-SWCNT/II-NCC; c) 3 SWCNT/II-NCC; and d) $2 a-S W C N T / I I-N C C$.

post-seeding. Thus, experiments with undifferentiated and differentiated cells (considered as cancer and normal cells, respectively) were performed between 2 and 5 days and 12-15 days post-seeding, respectively. Cells were seeded in 96-well plates at a density of $2 \times 10^{4}$ or $4 \times 10^{3}$ cells per well, and measurements were carried out 5 or 15 days after seeding, respectively. The culture medium was replaced with fresh medium (without FBS) containing the dispersions at the required concentrations, and with an exposure time of $72 \mathrm{~h}$.

\subsection{Cell viability assay}

Cell survival was measured using the MTT test. This assay is dependent on the cellular reduction of 3-(4,5-dimethylthiazol-2-yl)2,5-diphenyltetrazolium bromide (MTT, Sigma) by the mitochondrial dehydrogenase of viable cells to a blue formazan product that can be measured spectrophotometrically. Following appropriate incubation of the cells, with or without the samples, MTT $(5 \mathrm{mg} / \mathrm{mL})$ was added to each well in an amount equal to $10 \%$ of the culture volume. The cells were then incubated with MTT at $37^{\circ} \mathrm{C}$ for $3 \mathrm{~h}$. After that, the medium and MTT were removed, $100 \mu \mathrm{l}$ of DMSO was added to each well and the plate was gently stirred on a shaker. Finally, cell viability was determined by measuring the absorbance with a multi-well spectrophotometer (DTX 800 Multimode Detector, Beckman Coulter) at a wavelength of $560 \mathrm{~nm}$ and compared with the values for control cells incubated in the absence of the dispersions. Experiments were conducted in triplicate wells and repeated at least three times.

\subsection{Statistical analyses}

All results of cytotoxicity tests are expressed as means \pm the standard error of the mean (SEM) of at least three independent experiments. Statistical comparisons were performed using a one-way ANOVA followed by the Bonferroni post-test, and differences between P-values of $<0.05$ were considered to be statistically significant. Statistical analyses were carried out using the Prism GraphPad Program (Prism version 4.0, GraphPad Software, San Diego, CA).
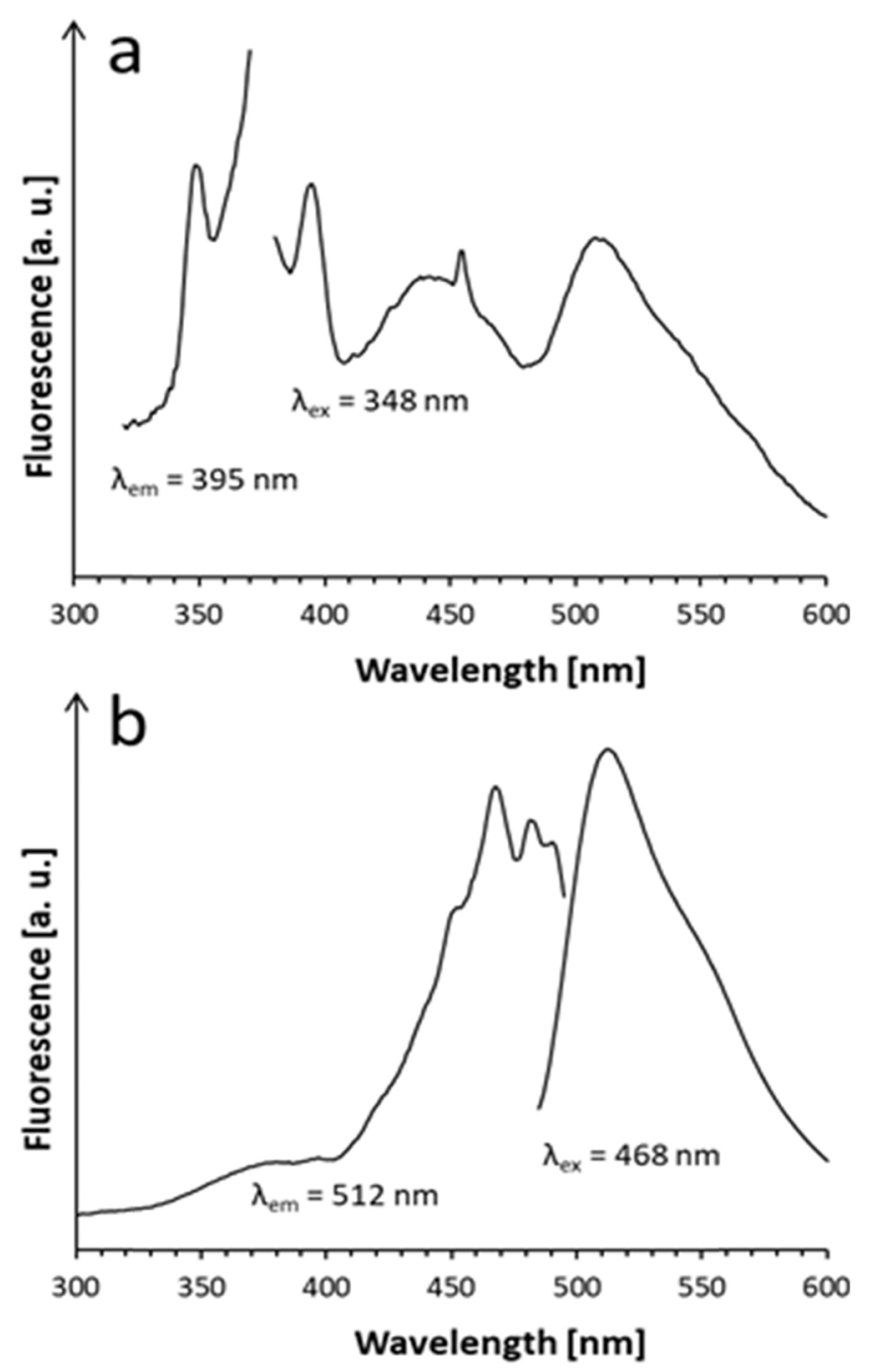

Fig. 4. Fluorescence spectra of the 3-SWCNT/II-NCC dispersion in water: a) excitation at $348 \mathrm{~nm}$; and b) excitation at $468 \mathrm{~nm}$.

\subsection{Stability tests}

In order to ascertain the stability of $f$-SWCNTs in the presence of biologically occurring reducing agents, some SWCNT/II-NCC hybrids were incubated in glutathione or ascorbic acid solutions in PBS. Either $2 a$-SWCNT or 3-SWCNT dispersed in type-II NCC was diluted in the biological solution $(10 \mathrm{mM}$ concentration of ascorbic acid or glutathione) to a final nanotube concentration of $30 \mu \mathrm{g} / \mathrm{mL}$ (the highest tested in all in vitro assays), together with vigorous vortex shaking and mild sonication. A control sample in PBS was also prepared for comparison purposes. These media were measured in terms of $\zeta$-potential, as stated above, at two dwelling times ( $2 \mathrm{~h}$ and $20 \mathrm{~h}$ ). In each case, the average of at least three independent measurements was used.

\subsection{Determination of SWCNT subcellular distribution}

The distribution of the different SWCNTs was evaluated by confocal microscopy. Confocal microscopy, by definition, generates high resolution images with depth selectivity, which allows us to differentiate fluorescent compounds inside and outside the cell, and even their subcellular localization. It is a standard technique for the study of dynamic internalization processes for drugs, nanomaterials, etc. We have characterized the internalization of nanomaterials in living cells using this technique in order to avoid the interference that may arise due to the 

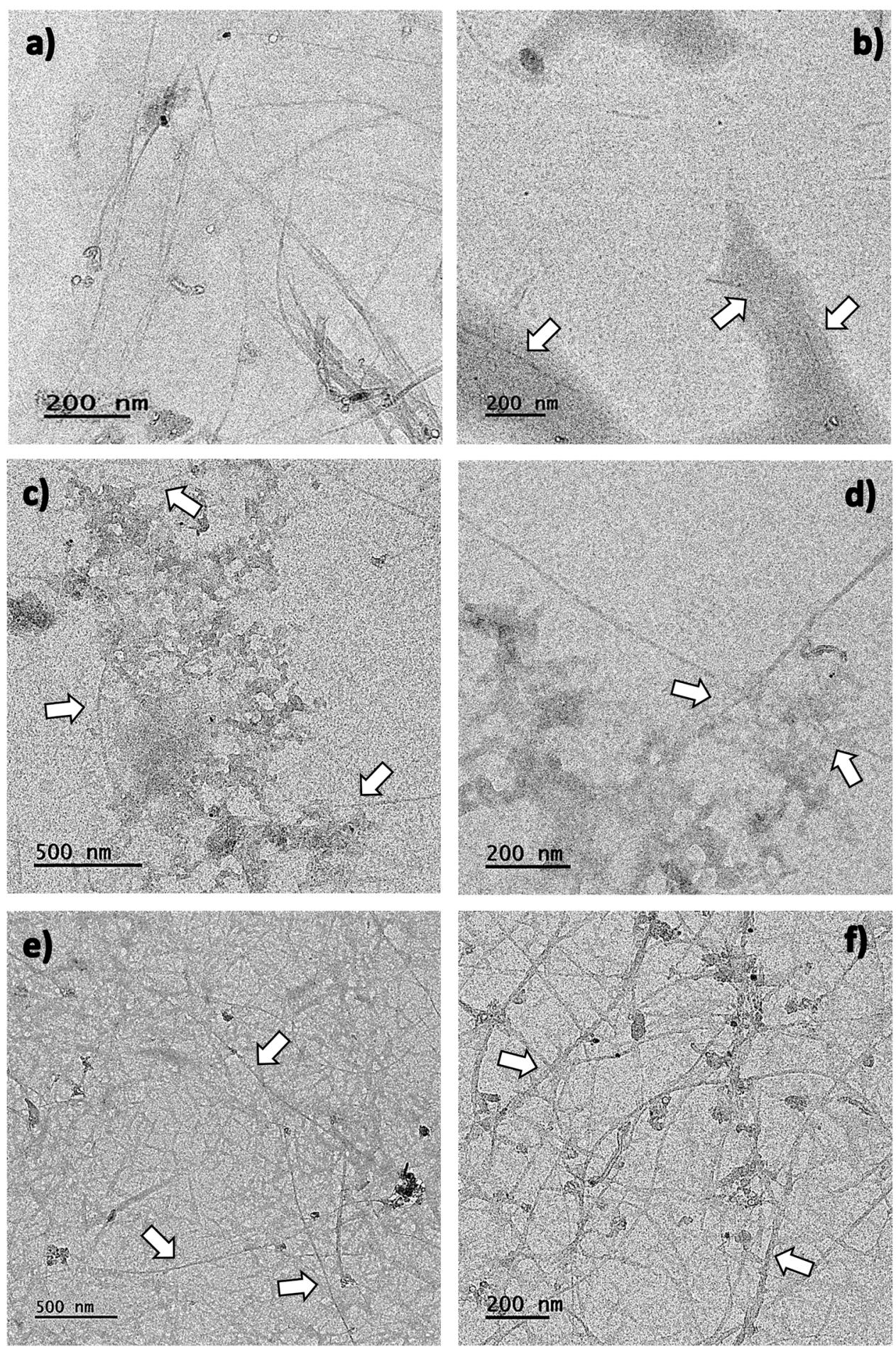

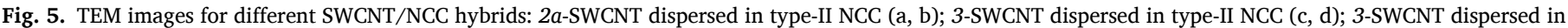
type-I NCC (e, f). White arrows denote the presence of some NCC-wrapped SWCNTs.

cell-fixation and -treatment processes required by the TEM and cryoTEM techniques, although at the expense of subcellular location accuracy. Briefly, $5 \mathrm{~d}$ and $14 \mathrm{~d}$ cells were treated with the different nanomaterials for 7 days then seeded in 96-well cell-imaging plates (\#0030741013; Eppendorf). Exposure to nanomaterials for $1 \mathrm{~h}$ was used as control. The cells were then incubated for 15 min with Mitotracker Red CMXRos (\# M7512; Thermo Fisher) and for $10 \mathrm{~min}$ with Hoescht 33342 ( $1 \mu \mathrm{g} / \mathrm{mL}$ ) (\#62249; Thermo Fisher), washed with Hanks solution and fresh medium, and imaged using a Zeiss LSM 880 inverted confocal microscope (40x and 63x objective).

\subsection{Evaluation of the mitochondrial fraction}

The effect of 1a-SWCNT, 2a-SWCNT and 3-SWCNT on the mitochondrial status was evaluated by confocal microscopy using Mitotracker Red CMXRos (\# M7512; Thermo Fisher). Briefly, 5d and 14d cells were treated for 7 days with the different nanomaterials and seeded in 96 well cell imaging plates (\#0030741013; Eppendorf). A 1-hour exposure to nanomaterials was used as a control. Cells were incubated for 15 min with Mitotracker Red CMXRos $(1 \mu \mathrm{M})$, washed in Hanks solution and fresh medium and imaged with a Zeiss LSM 880 inverted 


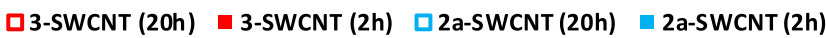

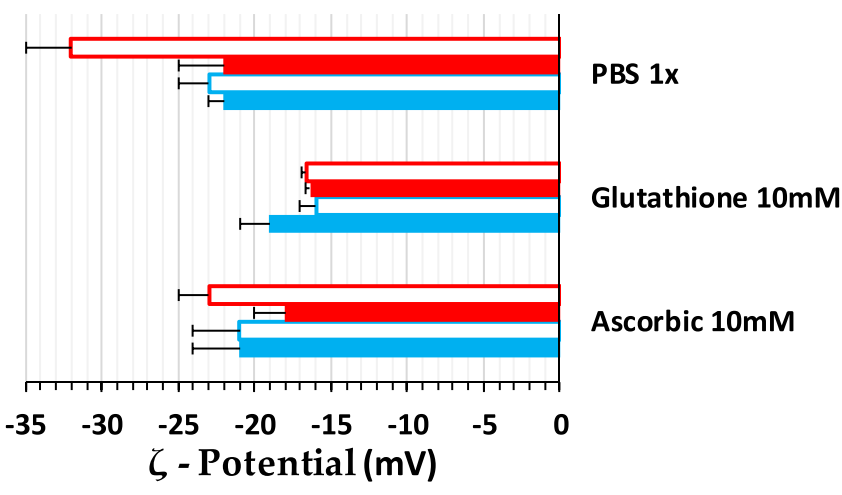

Fig. 6. Stability assessment of some SWCNT/II-NCC hybrids in different media throughout the $\zeta$-potential measurement.

confocal microscope $(20 \times$ objective). Mitotracker CMXRos levels were analyzed with ImageJ 1.53. Results showed the mean intensity (relative fluorescence units; RFUs) per cell ( $>75$ cells).

\section{Results and discussion}

\subsection{SWCNT functionalization}

The full functionalization picture is displayed in Scheme 1. Tracking of the functional groups incorporated into SWCNTs was performed using a combination of two techniques, namely TGA and Kaiser test (see experimental section). This allowed us to quantity covalently grafted moieties reasonably well. The thermal profiles of the main reaction products displayed in Fig. 1 show the typical change of behaviour from non-functionalized SWCNTs to their oxidized and functionalized counterparts. From the weight loss, and assuming the entire loss to be due to the incorporated moieties, the relative quantity of functional groups in each sample was easily estimated [35]. As additional support, in some samples in which benzylamine was deprotected and subsequently used for the anchoring of FL, the colorimetric Kaiser test provided quantitative information on the quantity of accessible primary amines, thus indirectly leading to quantification of FL functionalization. In the last stage of functionalization (sample 3-SWCNT), an additional characterization (by XPS) was applied in order to unambiguously determine the presence of capecitabine from the survey spectrum. This is possible due to the presence of fluorine atoms in its structure, which are clearly visible using this technique (Fig. 1, Table 1). Deconvoluted C1s, O1s and F1s core levels are presented in the Supplementary Materials (Fig. S1).

\subsection{Characterization of SWCNT/II-NCC dispersions}

After successful multiple functionalization of SWCNTs to generate a therapeutic vehicle with imaging and anticancer potential, our next goal was to enable a safe administration, for which a biocompatible dispersant was deemed necessary. In light of our previous results with NCC [36], hybrids of which with non-functionalized SWCNTs exhibited general biocompatibility and, in a specific case (with II-NCC), anti-colon cancer properties, we focused on ascertaining its suitability with functionalized SWCNTs. As a starting point, ox-SWCNTs were dispersed in water, and to that end, a comparison of NCC with several dispersing agents (DOC, GG, ALB, PEG, MCC) commonly used for similar purposes was undertaken in order to determine the aqueous stability with each dispersant. The quality and stability of the resulting colloidal dispersions was assessed by centrifugation and $\zeta$-potential measurements. The stability of $o x$-SWCNT/NCC hybrids against forced sedimentation was remarkable, even when compared with that achieved for the other surfactants (polymers, polysaccharides, and proteins; Fig. 2). The differential stabilization mechanism can be mostly associated with electrostatic phenomena since the centrifugation yield correlates well with the absolute value of the $\zeta$-potential (Fig. 2). Although the SWCNT hybrid with type I-NCC was more stable than the association with MCC and II-NCC, the SWCNT/II-NCC complex could be easily delivered in water suspension.

The concentration of SWCNT dispersions was monitored by Vis-NIR absorption spectroscopy. Typical spectra are shown in Fig. 3. The oxSWCNT dispersion exhibited bands at around 700 and $1000 \mathrm{~nm}$, which are associated with $\mathrm{M}_{11}$ and $\mathrm{S}_{22}$ electronic transitions in SWCNTs, respectively [34]. Both transition bands disappeared after covalent chemical functionalization via the radical arylation route, in agreement with many previous observations $[35,40,41]$. A weak band at around $450 \mathrm{~nm}$ in the functionalized samples indicated the presence of FL. The FL functional group is clearly tracked by fluorescence spectroscopy with SWCNT/NCC colloids (Fig. 4). Upon excitation at $348 \mathrm{~nm}$ (Fig. 4a), the emission peak at $395 \mathrm{~nm}$ is due to the SWCNTs, the band in the range $410-480 \mathrm{~nm}$ is due to the FA group linkage, a peak at $454 \mathrm{~nm}$ appears for the $\mathrm{CB}$ group, and the emission at $512 \mathrm{~nm}$ corresponds to FL. The typical spectral profile of FL is even more evident upon excitation at 468 nm (Fig. 4b).

The morphology of SWCNT/NCC hybrids was studied by TEM (Fig. 5). Both 2a-SWCNT and 3-SWCNT were visualized using this technique as the most relevant platforms. When dispersed in type-II NCC, the hybrids appear as SWCNTs encased by rod-shaped type-II nanocrystals (Fig. 5a-d). The size distribution and widths are in perfect agreement with a very efficient debundling and individualization of the SWCNTs due to the action of NCC. For comparison purposes, the 3SWCNT analogue with type-I NCC was also studied. In this case, although the size of the SWCNTs is similar, the morphology of the hybrid differs markedly, with the needle-like type-I nanocrystals being adsorbed on the surface of the SWCNTs. These observations are similar to those made in our previous study with similar SWCNTs [36].

The susceptibility of the different SWCNT/II-NCC hybrids to be reduced in intracellular environments was tested against biologically relevant reducing agents (glutathione and ascorbic acid), as an approximation of the stability in such media. Fig. 6 shows the $\zeta$-potential values after incubating different hybrids in these media at a SWCNT concentration of $30 \mu \mathrm{g} / \mathrm{mL}$, along with the stability in PBS for comparison. In this latter case, the $\zeta$-potential values are slightly lower at short times $(2 \mathrm{~h})$ but comparable to those exhibited by the ultracentrifuged SWCNT/II-NCC hybrids (Fig. 2). At $20 \mathrm{~h}$, and surprisingly, this stability increases for the 3-SWCNT hybrid and stays the same for its $2 a$-SWCNT counterpart. Given that PBS is the biologically preferred dispersion medium, it is clear that this medium does not cause any instability issues with the aqueous SWCNT/II-NCC suspensions, therefore the following observations are ascribable only to the reducing agents. A comparison of ascorbic acid with glutathione revealed that the hybrids are slightly more sensitive to the latter (reaching $\zeta$-potential values in the range of -16 to $-19 \mathrm{eV}$ ), with no significant differences with incubation time. The results in ascorbic acid are more similar to those for the PBS reference.

Taking into account that higher concentrations of dissolved salts in the CNT dispersion media generally lead to a lower $\zeta$-potential [42], and also that the $\zeta$-potential values tend to be very large at high CNT and dispersant concentrations [43], we can conclude that at such a high dilution as attained here ( $30 \mu \mathrm{g} / \mathrm{mL}$ of SWCNTs and $150 \mu \mathrm{g} / \mathrm{mL}$ of NCC) together with the highly electrolytic media, the studied hybrids show a fairly good colloidal stability under simulated intracellular conditions, above the agglomeration threshold $(\sim 10 \mathrm{eV}$ in absolute value), and exhibit an incipient stability as a result of negative charge repulsions. However, other stabilization mechanisms not seen using this technique (such as steric interactions) could also participate in the overall colloidal stability of the samples. 
a

II-NCC

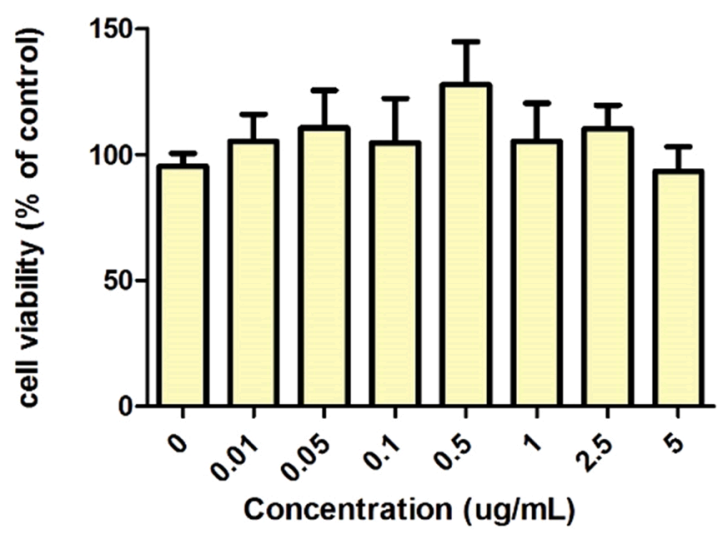

C

ox-SWCNT/II-NCC

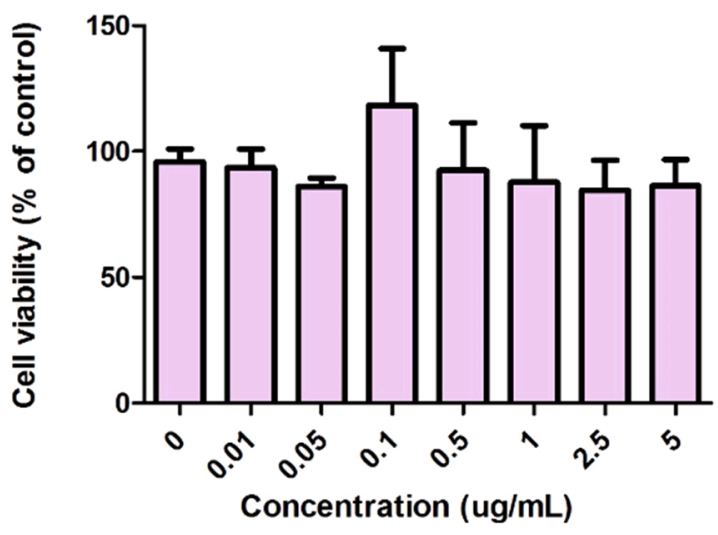

e

d-SWCNT/II-NCC

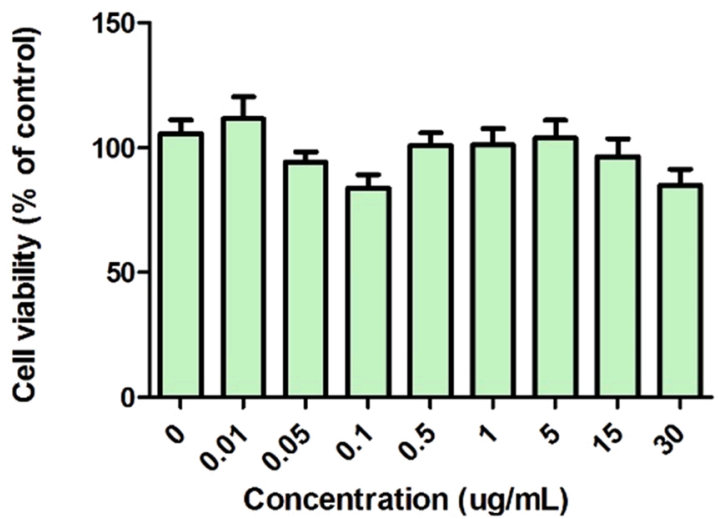

b

II-NCC

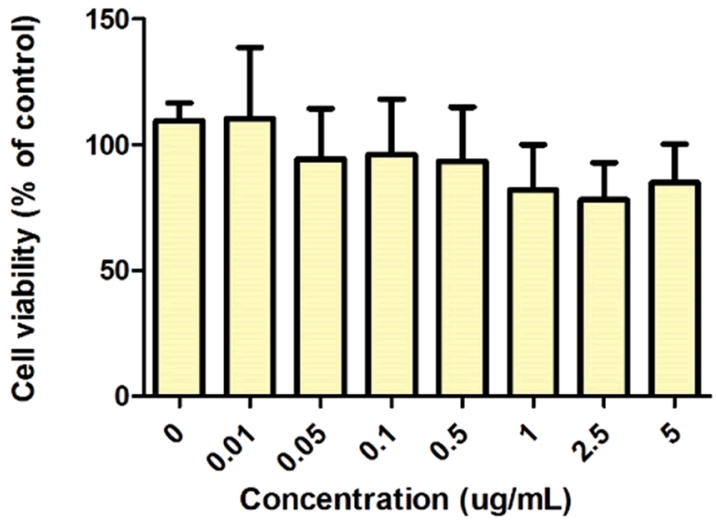

d

ox-SWCNT/II-NCC

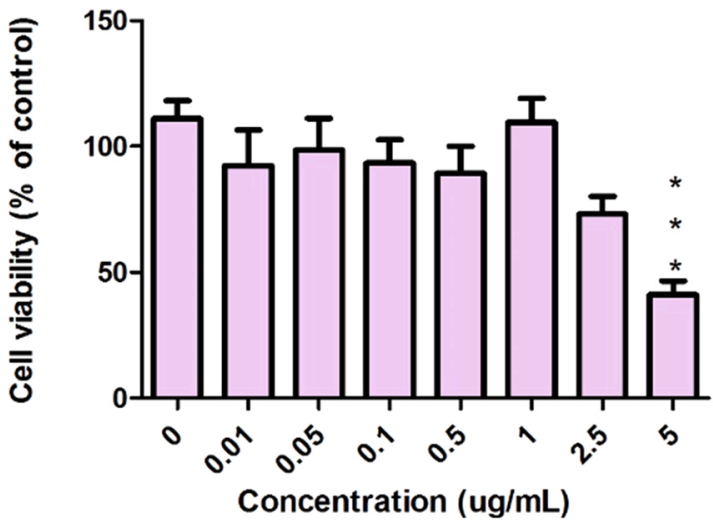

f

d-SWCNT/II-NCC

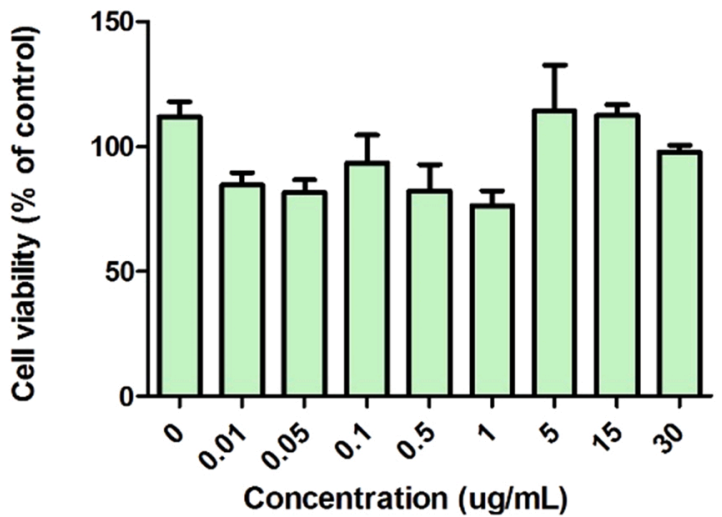

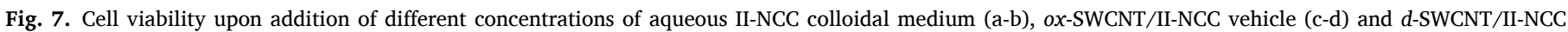
vehicle (e-f) in differentiated-normal cells (15 days culture, left column) and proliferative-cancer cells (5 days culture, right column). ***P $<0.001$ vs. control.

\subsection{Biocompatibility and bioactivity of SWCNT/II-NCC dispersions}

A non-toxic and generally innocuous behaviour of a therapeutic platform towards healthy cells and tissues is a required feature to ensure safe administration, targeting the cells responsible for a disease as specifically as possible. Both the active substance and the vehicle or medium have to fulfil this requirement. We have previously shown that air-oxidized SWCNTs dispersed in $5 \mathrm{mg} / \mathrm{mL}$ of Pluronic ${ }^{\circledR} \mathrm{F} 68$ block copolymer can induce an increase in intestinal contractility and oxidative stress [44]. In agreement with other research groups [23,24,45], we 
a

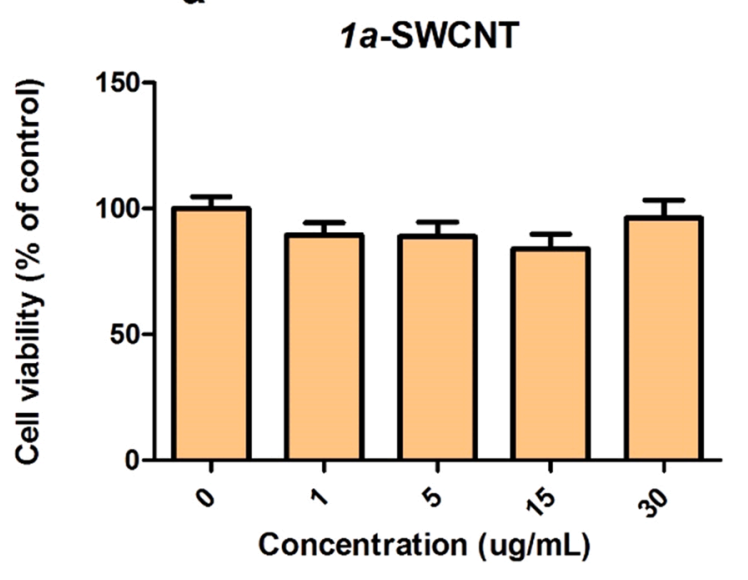

C

\section{3-SWCNT}

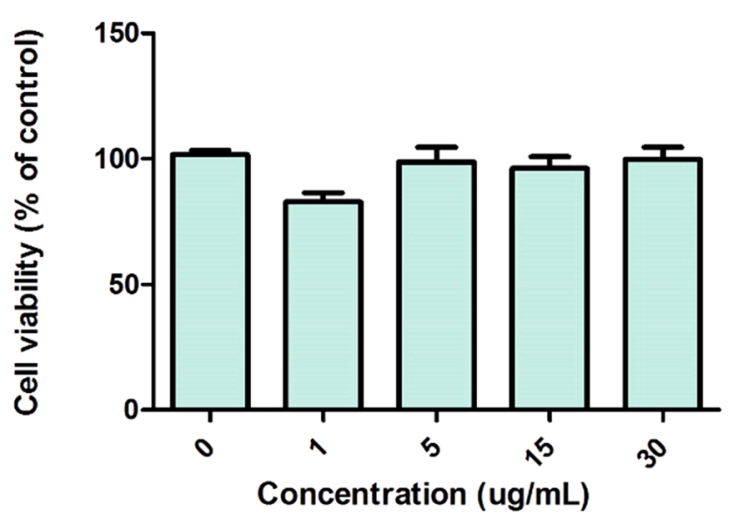

b

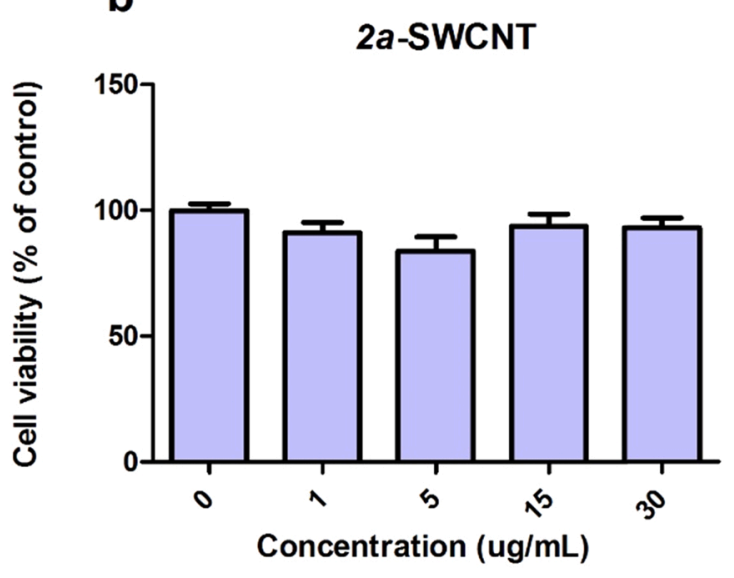

d

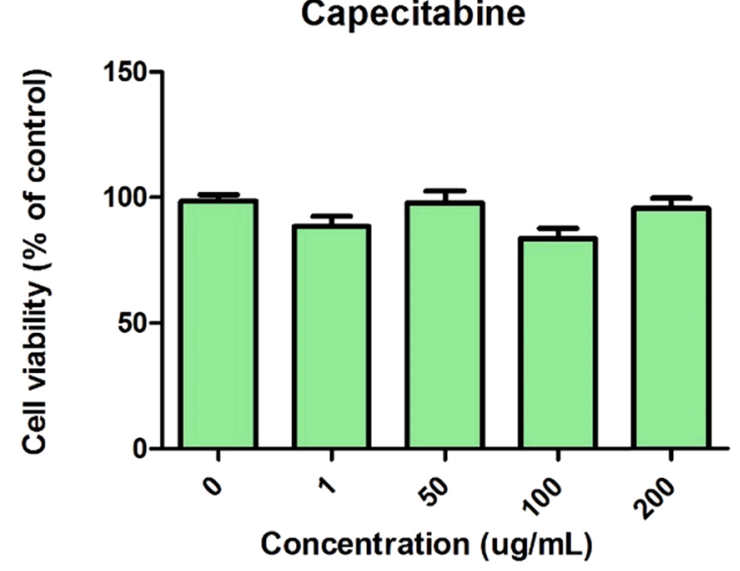

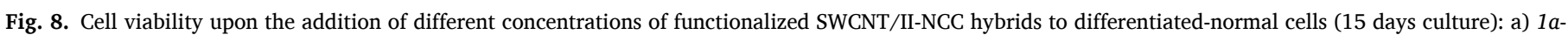
SWCNT; b) 2a-SWCNT; c) 3-SWCNT; and d) Capecitabine reference. No significant differences were observed.

noted that the toxicity could come from the dispersion medium even though it comprises molecules that can be present at low concentrations in biological environments, such as polylysine [46]. As such, particular attention is paid here to the aqueous dispersive medium, which can ultimately allow an oral or intravenous administration. More specifically, the oral route of administration is of great relevance in the treatment with chemotherapeutic compounds [47] and is a route of potential interest in the therapeutic use of nanomaterials [48]. Chen et al. demonstrated the presence of SWCNTs in the intestine of mice after oral exposure. In addition, they showed that low doses of SWCNTs did not generate pathological damage in the duodenum, or in the colon, and did not cause colitis-like symptoms [49]. All this indicates that the oral route would be of potential interest in future applications of our therapeutic agents.

The use of a bile salt such as DOC in the dispersion medium was considered first. However, the toxicity of $o x$-SWCNT/DOC suspensions was detected, in particular on normal cell cultures, clearly being attributable to the DOC surfactant (Fig. S2, Supplementary Materials). This dispersant was therefore immediately rejected. As regards other more biologically friendly dispersion media, such as PEG, GG, and ALB, no major toxicity effects were detected due to the dispersion agents alone. However, unexpected mortality and overproliferation events were observed for normal (differentiated) cells after the administration of centrifuged ox-SWCNT dispersions (Figs. S3e-S5e, Supplementary Materials).

Fig. 7 shows that II-NCC is non-toxic for in vitro cell cultures at the concentration required to disperse SWCNTs, but selective cancer cell killing is visible at $5 \mu \mathrm{g} / \mathrm{mL}$, in agreement with our previous results with both type-I and II-NCC [36]. Cytotoxicity tests remain negative for the $o x$-SWCNT/II-NCC and $d$-SWCNT/II-NCC dispersions with both normal (differentiated) and cancer (proliferative) cell cultures (Fig. 7), thus indicating that the dispersion media and drug vehicles used are generally innocuous.

The central findings and novelty of this work concern cytotoxicity tests of functionalized SWCNT/II-NCC hybrids with normal (differentiated) and cancer (proliferative) cell cultures (Figs. 8 and 9). The three hybrids with 1a-SWCNT, 2a-SWCNT and 3-SWCNT show no effects on normal cells, and cause remarkable decreases in the population of cancer cells. However, while this behavior is qualitatively analogous to the well-known reference drug capecitabine, the activity of SWCNT/II-NCC hybrids occurs at substantially lower mass concentrations.

As for the experiments related to the information displayed in Fig. 9, the potency of these potential anticancer platforms was assessed using the $\mathrm{IC}_{50}$ parameter, the half maximum inhibitory concentration towards Caco-2 cancer cells (Table 2). Our results show that the $\mathrm{IC}_{50}$ for $1 a$ SWCNT and $2 a$-SWCNT platforms is lower than for capecitabine itself, thus indicating that the designed platforms can be used to obtain the same anticancer effects but at a lower dose than the chemotherapeutic drug alone. When comparing these results with literature data, we found that the $\mathrm{IC}_{50}$ of capecitabine in different colorectal cell lines, such as LS174T or Caco-2, is 300 and $80 \mu \mathrm{g} / \mathrm{mL}$, respectively [50,51], much higher than the values obtained for the $\mathrm{IC}_{50}$ of capecitabine in our study in Caco- 2 cells.

The selective activity against cancer (proliferative) cells is identical using 1a-SWCNT and 3-SWCNT, thus indicating that the bonded capecitabine group is not relevant. In contrast, the activity observed is 
a

1a-SWCNT

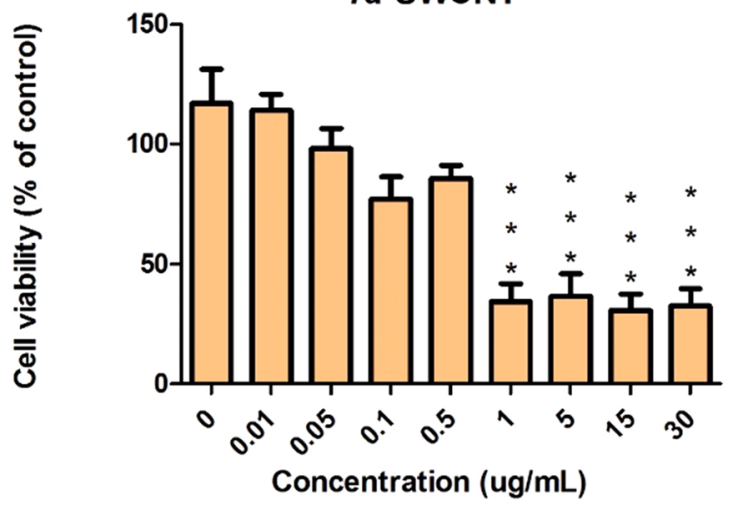

C

3-SWCNT

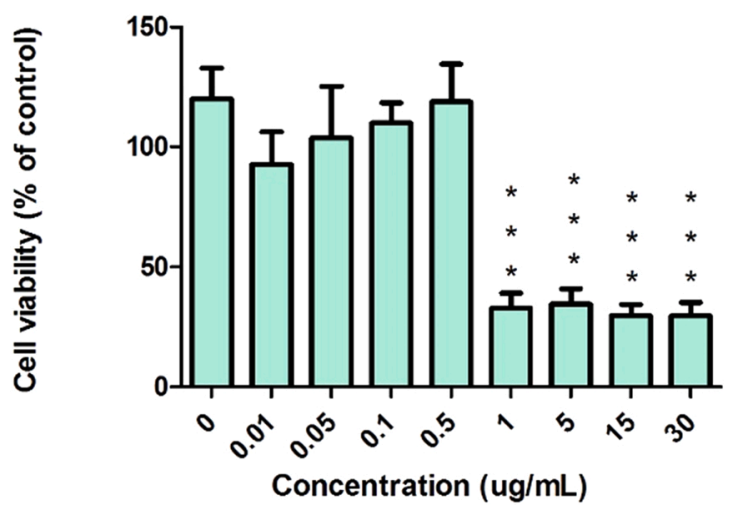

b

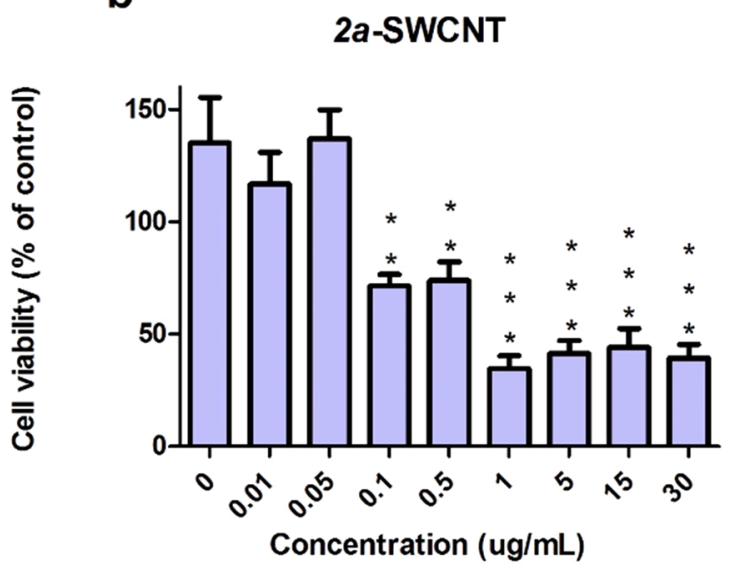

d

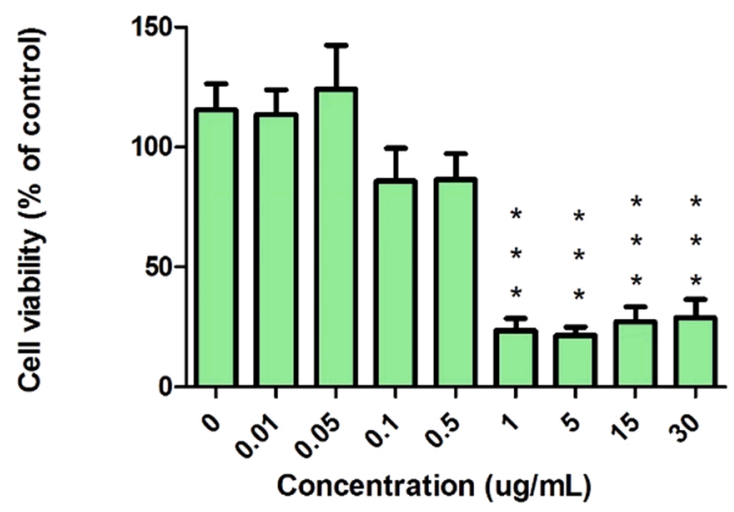

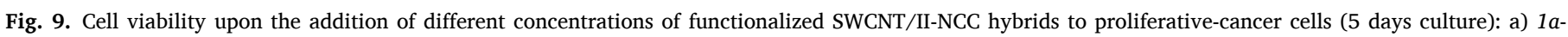
SWCNT; b) 2a-SWCNT; c) 3-SWCNT; and d) Capecitabine reference. ${ }^{*}{ }^{*} \mathrm{P}<0.01,{ }^{*} * * \mathrm{P}<0.001$ vs. control.

Table 2

IC $_{50}$ values for the different SWCNT (dispersed in type-II NCC) platforms in Caco-2 cancer cells. The reference drug capecitabine without any additive is also given for comparison. The results are expressed as mean values $\pm \log$ of the standard deviation of the mean ( $n \geq 12$ experiments).

\begin{tabular}{ll}
\hline Platform & $\mathrm{IC}_{50}(\mu \mathrm{g} / \mathrm{mL})$ \\
\hline 1a-SWCNT & $0.201 \pm 0.115$ \\
2a-SWCNT & $0.113 \pm 0.115$ \\
3-SWCNT & $0.617 \pm 0.151$ \\
Capecitabine (alone) & $0.279 \pm 0.110$ \\
\hline
\end{tabular}

apparently caused by the joint action of SWCNTs with the FL group stabilized in II-NCC. Moreover, the activity can be enhanced by the FA targeting group, as shown by the results of delivering $2 a$-SWCNT (Fig. 9). In a reference study carried out by Ménard-Moyon and coworkers [52] using a functionalized CNT vehicle triply functionalized with FL, FA and the drug gemcitabine, the authors found a low specificity of the FA functional group covalently anchored to CNTs towards both folate-positive and folate-negative cancer cell lines. This was explained as being due to a combination of alternative internalization routes, with a higher sensitivity of the folate-negative cell line towards the tested drug. Our selective effect against cancer cells is not observed if the folate group is attached to SWCNTs in the absence of FL (sample $2 b$ '-SWCNT, Fig. 10), thereby agreeing with Ménard-Moyon's observations and suggesting that the internalization of CNTs may not depend critically on the folate receptors. In contrast to this reference work [52], we did not observe an actual effect of the drug covalently linked to our SWCNTs, however it is difficult to make a direct comparison as the type and origin of the CNTs are different, as are the cell lines and the type of in vitro assay. In essence, given the broad range of possibilities offered by CNTs and their potential for multifunctionalization, herein we report a new outcome, mainly stemming from the use of NCC as a biocompatible and bioactive adjuvant for CNTs, and the boosting of these effects by specific functionalization.

Moreover, our hybrids are not active if type-I NCC is utilized as the dispersing agent (Fig. 10). We have recently reported this unique selectivity of SWCNT/II-NCC hybrids against colon cancer cells with non-oxidized and non-functionalized SWCNTs, while type-I NCC hybrids are not active [36]. We have also observed a selective effect using aqueous solutions of certain functionalized carbon nanoparticles [53]. However, the selective activity reported here is much stronger and is a direct consequence of specific functionalization at the SWCNT surface.

It is worth noting at this point that the use of common indirect in vitro assays to assess cell viability is debatable in the case of CNTs. In particular, the MTT assay has been questioned as formazan could be sequestered from the medium by adsorption on the CNT sidewalls and cells might also uptake large quantities of CNTs whose intrinsic absorbance could cause interference when measuring at $570 \mathrm{~nm}$ [45]. This would not be the case here, at least for the NCC dispersants, because the high and efficient degree of SWCNT sidewall coverage by NCC was verified by TEM and also in a previous study [36], therefore the interaction of formazan with the sidewalls is very unlikely. The low SWCNT uptake observed by confocal fluorescence spectroscopy (vide infra) suggests no interference either. Therefore, we have developed a stable 
a

3-SWCNT/NCC(type I)

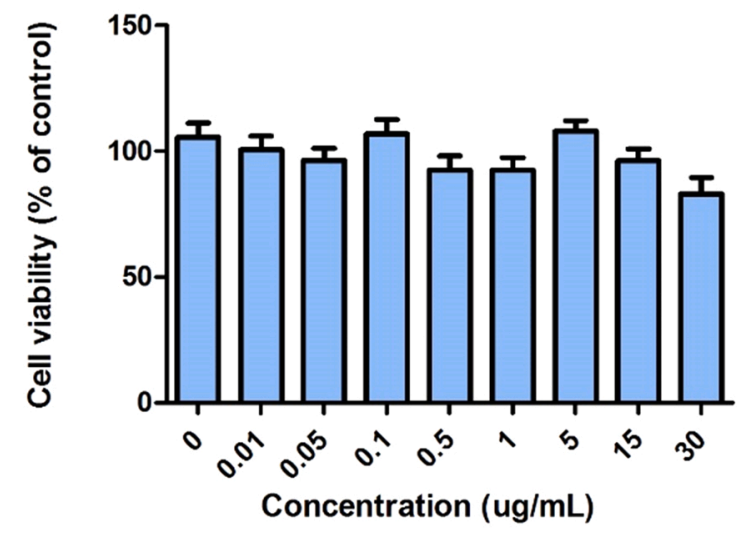

C

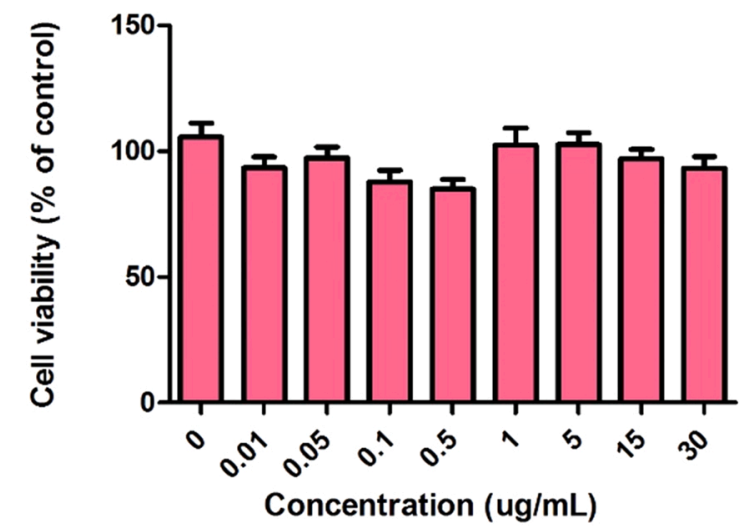

b

3-SWCNT/NCC (type I)

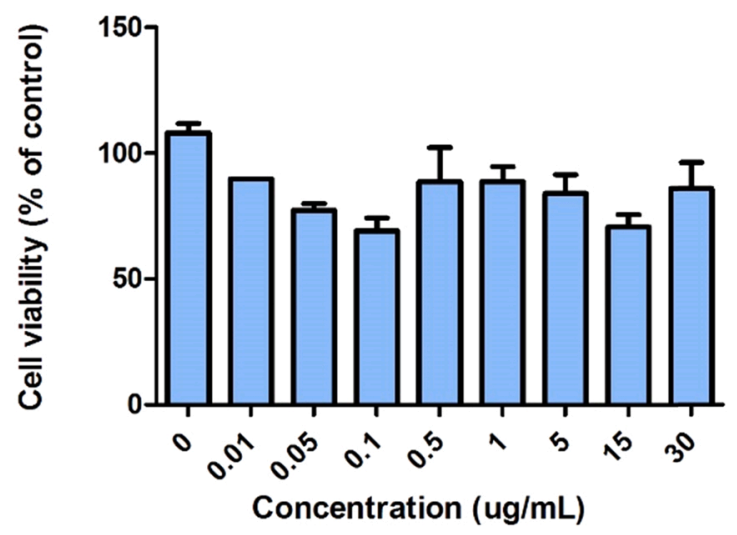

d

$2 b-S W C N T / I I-N C C$

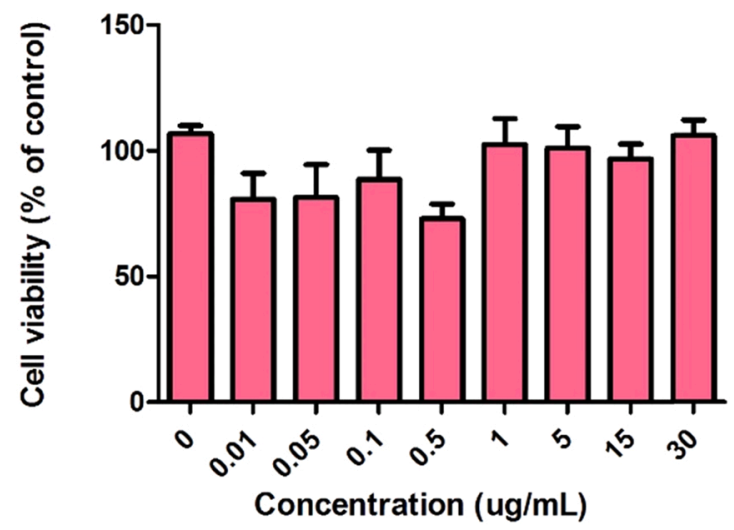

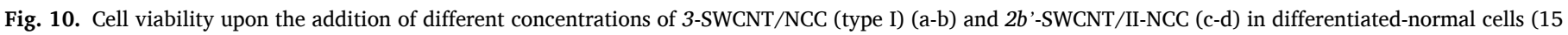
days culture, left column) and proliferative-cancer cells (5 days culture, right column). No significant differences were observed.

and effective chemotherapeutic agent, based on the SWCNT/II-NCC hybrid platform, with potential for selectively targeting colon cancer cells and the possibility of being enhanced by covalent chemical functionalization.

Finally, we should stress the relevance of being able to image the active hybrid complex by fluorescence [54]. Confocal microscopy images (Fig. 11) clearly show that the presence of functionalized SWCNTs in cell cultures can be detected using the fluorescein chemical group. Using this method, we checked the internalization of the different SWCNTs after $72 \mathrm{~h}$ of exposure and found that this occurred in both cell phenotypes (Fig. 11, $72 \mathrm{~h}$ ). As such, this technique allows us to monitor the subcellular location of the nanomaterials. The three-dimensional z-stack representation shows the different internalised SWCNTs in greater detail (Fig. S6a-d). The position and location of 3 different 1a-SWCNTs can be observed in different three-dimensional projections (Fig. S6c-d). No internalization was observed after short-term exposure (Fig. 11, $1 \mathrm{~h}$ ). In a first approach, we observed how SWCNTs interacted with mitochondria and the cell nucleus, mainly being located in the cell nucleus. We did not notice any differences in the internalization or subcellular location of SWCNTs in the different cellular phenotypes. Interestingly, using this approach, we observed an alteration in mitotracker intensity that can be associated with changes in mitochondrial activity. We analyzed the level of Mitotracker CMXRos intensity, observing a significant decrease in signal intensity (relative fluorescence units, RFUs) in proliferative-cancer cells treated with the different SWCNTs $(1 \mu \mathrm{g} / \mathrm{mL})$ (Fig. S7a, Supplementary Materials). These results point to a decrease in viability, validating previously reported results.
On the other hand, in differentiated-normal cells, we observed a significant increase in mitochondrial function (Fig. S7b, Supplementary Materials). These results show how the SWCNTs internalization influences mitochondrial function ultimately affecting cell viability. The improvement of mitochondrial function observed in differentiated cells suggests a potential beneficial effect in non-tumor cells, which will be further studied.

However, quantitative studies are still required to determine the subcellular location with certainty, as well as to determine the cell internalization mechanism involved. Herein the aim was to provide an overview of this internalization, which was observed in several cells in the same field of view. This contrasts with electron microscopy techniques, the high resolution of which limits the number of cells that can be observed in the same field. By using this technique, we consider all biases to be eliminated, thus providing an overall perspective.

\section{Conclusion}

Functionalized SWCNTs are successfully stabilized in water dispersion by NCC, with the resulting hybrids showing no toxicity, in contrast to surfactants such as DOC, and a stabilization ability equivalent to other polymers and biomolecules, such as PEG, GG, and ALB. Aqueous dispersions of fluorescein-functionalized SWCNTs in type II-NCC show an increase in the intrinsic activity of the hybrid against colon cancer cells compared to the non-functionalized counterpart and the reference drug capecitabine, and are also non-toxic for normal colon cells. Functionalization with the folic acid targeting agent promotes the activity of this 

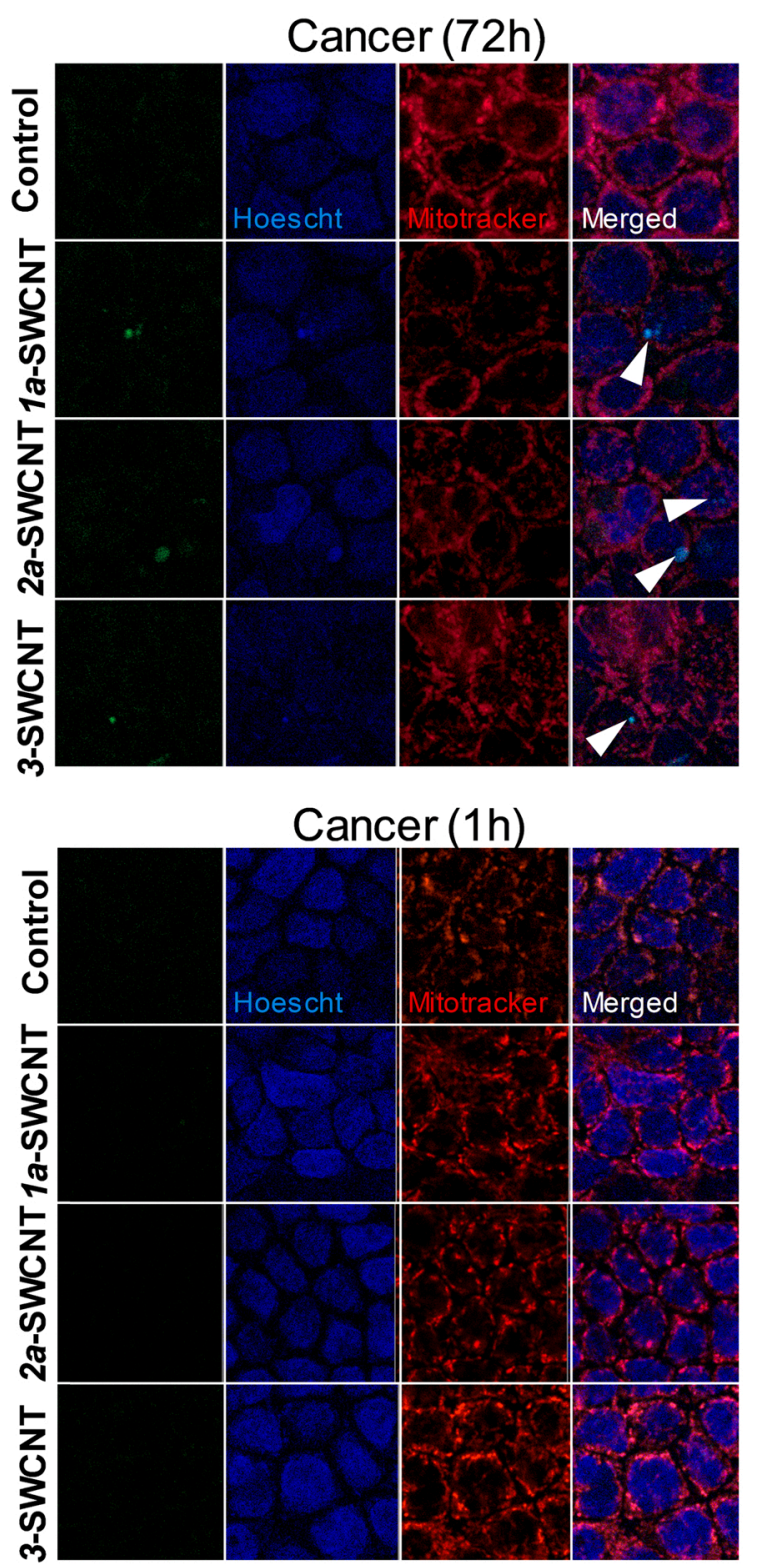
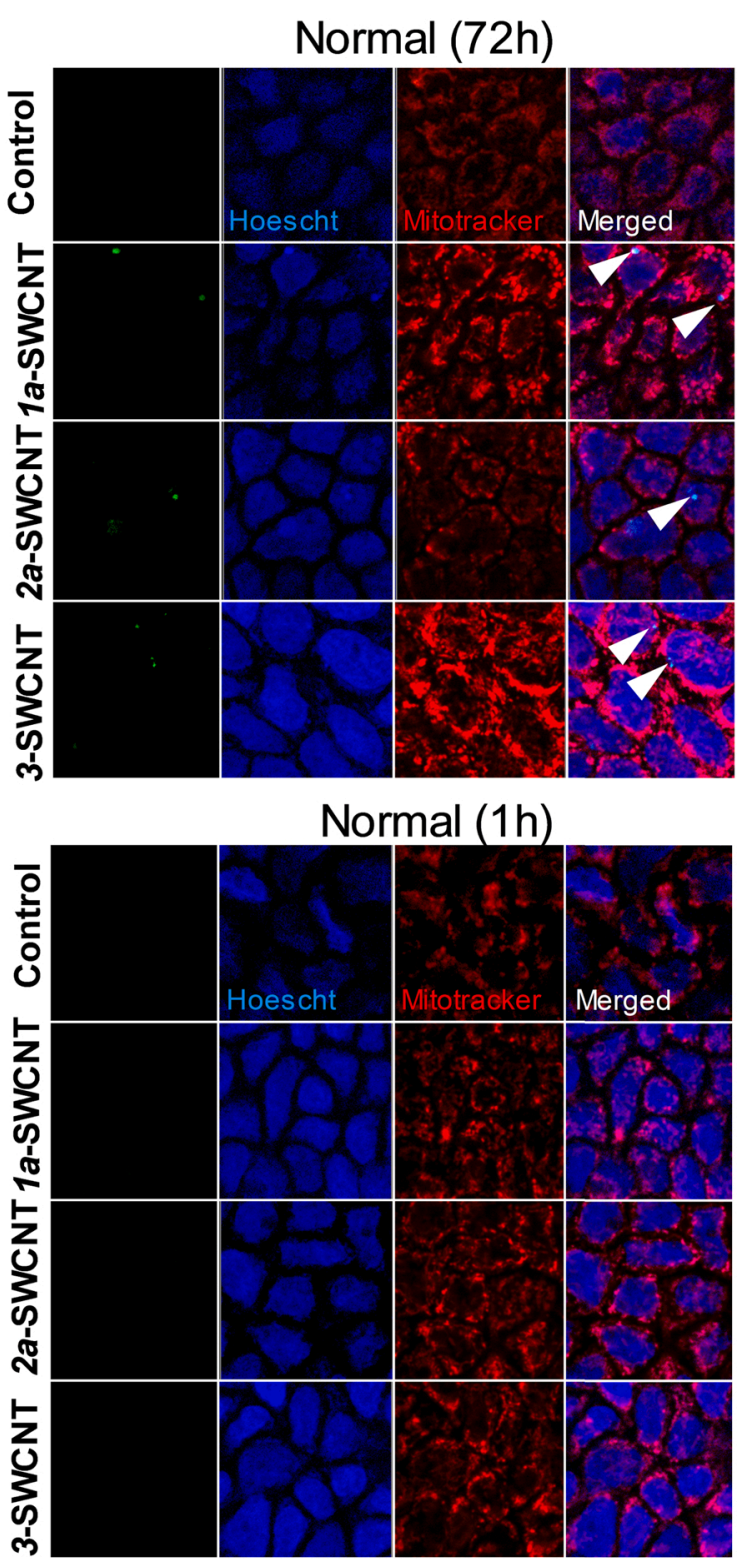

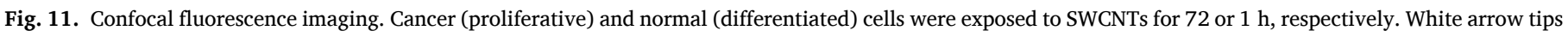
highlight the presence of the SWCNT fluorescence in the merged images.

platform against cancer cells. However, an additional functionalization with capecitabine does not provide any further response. An analogous complex with type-I NCC is not active either. This latter result suggests caution when the mechanisms of action of multifunctionalized nanoparticulate drug carriers are proposed. Here, the $2 a$-SWCNT/II-NCC complex is proposed as a new chemotherapeutic platform that can be also used as a theranostic agent by virtue of its imaging capability in common medical techniques, such as fluorescence at green wavelengths.

\section{CRediT authorship contribution statement}

J.M. González-Domínguez: Conceptualization, Methodology, Software, Formal analysis, Resources, Writing - original draft, Writing review \& editing, Supervision. L. Grasa: Conceptualization, Methodology, Software, Formal analysis, Resources, Data curation, Writing - review \& editing. J. Frontiñán-Rubio: Methodology, Software, Formal analysis, Investigation, Data curation, Writing - review \& editing. E. Abás: Methodology, Software, Formal analysis, Investigation, Data curation. A. Domínguez-Alfaro: Investigation, Visualization. J. E.
Mesonero: Validation, Resources, Writing - review \& editing. A. Criado: Software, Investigation, Visualization. A. Ansón-Casaos: Conceptualization, Methodology, Formal analysis, Investigation, Resources, Writing - original draft.

\section{Declaration of Competing Interest}

The authors declare that they have no known competing financial interests or personal relationships that could have appeared to influence the work reported in this paper.

\section{Acknowledgements}

This research was funded by the regional government of Aragón, DGA (Grupos Reconocidos DGA-T03_17R, DGA-T03_20R and DGAA20_20R), together with associated EU Regional Development Funds, and also the Spanish MINEICO through a "Juan de la Cierva Incorporación" contract, and their associated research funds (ref. IJCI2016-27789). A.C. thanks the Xunta de Galicia for an "Atracción de 
Talento" research grant (no. ED431H 2020/17).

\section{Appendix A. Supporting information}

Supplementary data associated with this article can be found in the online version at doi:10.1016/j.colsurfb.2022.112363.

\section{References}

[1] A.Z. Juthi, M. Aquib, M.A. Farooq, S. Ghayas, F. Khalid, G.F. Boafo, D.P. Wande, D H. Khan, T.Z. Bithi, R. Bavi, B. Wang, Theranostic applications of smart nanomedicines for tumor-targeted chemotherapy: a review, Environ. Chem. Lett. 18 (2020) 1509-1527, https://doi.org/10.1007/s10311-020-01031-8.

[2] Y. Zhu, N. Xin, Z. Qiao, S. Chen, L. Zeng, Y. Zhang, D. Wei, J. Sun, H. Fan, Bioactive MOFs Based Theranostic Agent for Highly Effective Combination of Multimodal Imaging and Chemo-Phototherapy, in: Adv. Healthc. Mater, 9, 2020, https://doi. org/10.1002/adhm.202000205.

[3] S. Dhar, Z. Liu, J. Thomale, H. Dai, S.J. Lippard, Targeted single-wall carbon nanotube-mediated $\mathrm{Pt}(\mathrm{IV})$ prodrug delivery using folate as a homing device, J. Am. Chem. Soc. 130 (2008) 11467-11476, https://doi.org/10.1021/ja803036e.

4] P. Li, Y. Wang, F. Zeng, L. Chen, Z. Peng, L.X. Kong, Synthesis and characterization of folate conjugated chitosan and cellular uptake of its nanoparticles in HT-29 cells, Carbohydr. Res. 346 (2011) 801-806, https://doi.org/10.1016/j. carres.2011.01.027.

[5] M. Thakur, A. Mewada, S. Pandey, M. Bhori, K. Singh, M. Sharon, M. Sharon, Milkderived multi-fluorescent graphene quantum dot-based cancer theranostic system, Mater. Sci. Eng. C. 67 (2016) 468-477, https://doi.org/10.1016/j. msec. 2016.05.007.

[6] S. Li, S. Zhou, Y. Li, X. Li, J. Zhu, L. Fan, S. Yang, Exceptionally high payload of the IR780 iodide on folic acid-functionalized graphene quantum dots for targeted photothermal therapy, ACS Appl. Mater. Interfaces 9 (2017) 22332-22341, https://doi.org/10.1021/acsami.7b07267.

[7] P. Gong, L. Sun, F. Wang, X. Liu, Z. Yan, M. Wang, L. Zhang, Z. Tian, Z. Liu, J. You, Highly fluorescent $\mathrm{N}$-doped carbon dots with two-photon emission for ultrasensitive detection of tumor marker and visual monitor anticancer drug loading and delivery, Chem. Eng. J. 356 (2019) 994-1002, https://doi.org/ 10.1016/j.cej.2018.09.100.

[8] X. Ji, H. Lv, X. Sun, C. Ding, Green-emitting carbon dot loaded silica nanoparticles coated with DNA-cross-linked hydrogels for sensitive carcinoembryonic antigen detection and effective targeted cancer therapy, Chem. Commun. 55 (2019) 15101-15104, https://doi.org/10.1039/C9CC07831B.

[9] H. Zhao, Y. Chao, J. Liu, J. Huang, J. Pan, W. Guo, J. Wu, M. Sheng, K. Yang, J. Wang, Z. Liu, Polydopamine coated single-walled carbon nanotubes as a versatile platform with radionuclide labeling for multimodal tumor imaging and therapy, Theranostics 6 (2016) 1833-1843, https://doi.org/10.7150/thno.16047.

[10] S. Wang, Q. Zhang, P. Yang, X. Yu, L.-Y. Huang, S. Shen, S. Cai, Manganese oxidecoated carbon nanotubes as dual-modality lymph mapping agents for photothermal therapy of tumor metastasis, ACS Appl. Mater. Interfaces 8 (2016) 3736-3743, https://doi.org/10.1021/acsami.5b08087.

[11] L. Fétiveau, G. Paul, A. Nicolas-Boluda, J. Volatron, R. George, S. Laurent, R. Muller, L. Sancey, P. Mejanelle, A. Gloter, F. Gazeau, L. Catala, Tailored ultrasmall Prussian blue-based nanoparticles for MRI imaging and combined photothermal/photoacoustic theranostics, Chem. Commun. 55 (2019) 14844-14847, https://doi.org/10.1039/C9CC07116D.

[12] L. Zhou, Y. Jing, Y. Liu, Z. Liu, D. Gao, H. Chen, W. Song, T. Wang, X. Fang, W. Qin, Z. Yuan, S. Dai, Z.A. Qiao, C. Wu, Mesoporous carbon nanospheres as a multifunctional carrier for cancer theranostics, Theranostics 8 (2018) 663-675, https://doi.org/10.7150/thno.21927.

[13] J.T.W. Wang, R. Klippstein, M. Martincic, E. Pach, R. Feldman, M. Šefl, Y. Michel, D. Asker, J.K. Sosabowski, M. Kalbac, T. Da Ros, C. Ménard-Moyon, A. Bianco, I. Kyriakou, D. Emfietzoglou, J.-C. Saccavini, B. Ballesteros, K.T. Al-Jamal, G. Tobias, Neutron activated $153 \mathrm{Sm}$ sealed in carbon nanocapsules for in vivo imaging and tumor radiotherapy, ACS Nano 14 (2020) 129-141, https://doi.org/ 10.1021/acsnano.9b04898.

[14] C. Liang, S. Diao, C. Wang, H. Gong, T. Liu, G. Hong, X. Shi, H. Dai, Z. Liu, Tumor metastasis inhibition by imaging-guided photothermal therapy with single-walled carbon nanotubes, Adv. Mater. 26 (2014) 5646-5652, https://doi.org/10.1002/ adma.201401825.

[15] X. Zhang, L. Meng, Q. Lu, Z. Fei, P.J. Dyson, Targeted delivery and controlled release of doxorubicin to cancer cells using modified single wall carbon nanotubes, Biomaterials 30 (2009) 6041-6047, https://doi.org/10.1016/j. biomaterials.2009.07.025.

[16] H. Huang, Q. Yuan, J.S. Shah, R.D.K. Misra, A new family of folate-decorated and carbon nanotube-mediated drug delivery system: synthesis and drug delivery response, Adv. Drug Deliv. Rev. 63 (2011) 1332-1339, https://doi.org/10.1016/j. addr.2011.04.001.

[17] X. Zhang, Q. Ba, Z. Gu, D. Guo, Y. Zhou, Y. Xu, H. Wang, D. Ye, H. Liu, Fluorescent coumarin-artemisinin conjugates as mitochondria-targeting theranostic probes for enhanced anticancer activities, Chem. Eur. J. 21 (2015) 17415-17421, https://doi. org/10.1002/chem.201502543.

[18] K.V. Sudheesh, P.S. Jayaram, A. Samanta, K.S. Bejoymohandas, R.S. Jayasree, A. Ajayaghosh, A cyclometalated iriii complex as a lysosome-targeted photodynamic therapeutic agent for integrated imaging and therapy in cancer cells, Chem. Eur. J. 24 (2018) 10999-11007, https://doi.org/10.1002/ chem. 201801918.

[19] G. Ficociello, A. Salemme, D. Uccelletti, S. Fiorito, A.R. Togna, L. Vallan, J. M. González-Domínguez, T. Da Ros, S. Francisci, A. Montanari, Evaluation of the efficacy of carbon nanotubes for delivering peptides into mitochondria, RSC Adv. 6 (2016) 67232-67241, https://doi.org/10.1039/C6RA14254K.

[20] S. Boncel, A. Pluta, M. Skonieczna, A. Gondela, B. Maciejewska, A.P. Herman, R. G. Jedrysiak, S. Budniok, K. Komedera, A. Błachowski, K.Z. Walczak, Hybrids of iron-filled multiwall carbon nanotubes and anticancer agents as potential magnetic drug delivery systems: in vitro studies against human melanoma, colon carcinoma, and colon adenocarcinoma, J. Nanomater. (2017), 1262309, https://doi.org/ $10.1155 / 2017 / 1262309$.

[21] M. Sancho-Albero, B. Rubio-Ruiz, A.M. Pérez-López, V. Sebastián, P. Martín Duque, M. Arruebo, J. Santamaría, A. Unciti-Broceta, Cancer-derived exosomes loaded with ultrathin palladium nanosheets for targeted bioorthogonal catalysis, Nat. Catal. 2 (2019) 864-872, https://doi.org/10.1038/s41929-019-0333-4.

[22] B. Dinesh, A. Bianco, C. Ménard-Moyon, Designing multimodal carbon nanotubes by covalent multi-functionalization, Nanoscale 8 (2016) 18596-18611, https:// doi.org/10.1039/C6NR06728J.

[23] L. Dong, K.L. Joseph, C.M. Witkowski, M.M. Craig, Cytotoxicity of single-walled carbon nanotubes suspended in various surfactants, Nanotechnology 19 (2008), 255702, https://doi.org/10.1088/0957-4484/19/25/255702.

[24] A.L. Alpatova, W. Shan, P. Babica, B.L. Upham, A.R. Rogensues, S.J. Masten, E. Drown, A.K. Mohanty, E.C. Alocilja, V.V. Tarabara, Single-walled carbon nanotubes dispersed in aqueous media via non-covalent functionalization: effect of dispersant on the stability, cytotoxicity, and epigenetic toxicity of nanotube suspensions, Water Res. 44 (2010) 505-520, https://doi.org/10.1016/j. watres.2009.09.042.

[25] J.M. González-Domínguez, M.A. Álvarez-Sánchez, C. Hadad, A.M. Benito, W. K. Maser, in: N. Martín, T. Da Ros, J.F. Nierengarten (Eds.), 1st edn..,Carbon Nanostructures for Biomedical Applications, ch. 4, Royal Society of Chemistry, Cambridge (UK), 2021, pp. 98-152.

[26] J.B. Mougel, C. Adda, P. Bertoncini, I. Capron, B. Cathala, O. Chauvet, Highly efficient and predictable noncovalent dispersion of single-walled and multi-walled carbon nanotubes by cellulose nanocrystals, J. Phys. Chem. C. 120 (2016) 22694-22701, https://doi.org/10.1021/acs.jpcc.6b07289.

[27] A. Hajian, S.B. Lindström, T. Pettersson, M.M. Hamedi, L. Wågberg, Understanding the dispersive action of nanocellulose for carbon nanomaterials, Nano Lett. 17 (2017) 1439-1447, https://doi.org/10.1021/acs.nanolett.6b04405.

[28] B.A. Cisterna, N. Kamaly, W. Il Choi, A. Tavakkoli, O.C. Farokhzad, C. Vilos, Targeted nanoparticles for colorectal cancer, Nanomedicine 11 (2016) 2443-2456, https://doi.org/10.2217/nnm-2016-0194.

[29] X. You, Y. Kang, G. Hollett, X. Chen, W. Zhao, Z. Gu, J. Wu, Polymeric nanoparticles for colon cancer therapy: overview and perspectives, J. Mater. Chem. B 4 (2016) 7779-7792, https://doi.org/10.1039/C6TB01925K.

[30] X. Fan, G. Jiao, L. Gao, P. Jin, X. Li, The preparation and drug delivery of a graphene-carbon nanotube- $\mathrm{Fe}_{3} \mathrm{O}_{4}$ nanoparticle hybrid, J. Mater. Chem. B 1 (2013) 2658, https://doi.org/10.1039/c3tb00493g.

[31] C. Tripisciano, M.H. Rümmeli, X. Chen, E. Borowiak-Palen, Multi-wall carbon nanotubes - a vehicle for targeted Irinotecan drug delivery, Phys. Status Solidi B 247 (2010) 2673-2677, https://doi.org/10.1002/pssb.201000143.

[32] M. Zhou, Z. Peng, S. Liao, P. Li, S. Li, Design of microencapsulated carbon nanotube-based microspheres and its application in colon targeted drug delivery, Drug Deliv. 21 (2014) 101-109, https://doi.org/10.3109/10717544.2013.834413.

[33] K. Wei, X. Peng, F. Zou, Folate-decorated PEG-PLGA nanoparticles with silica shells for capecitabine controlled and targeted delivery, Int. J. Pharm. 464 (2014) 225-233, https://doi.org/10.1016/j.ijpharm.2013.12.047.

[34] A. Ansón-Casaos, J.M. González-Domínguez, I. Lafragüeta, J.A. Carrodeguas, M. T. Martínez, Optical absorption response of chemically modified single-walled carbon nanotubes upon ultracentrifugation in various dispersants, Carbon 66 (2014) 105-118, https://doi.org/10.1016/j.carbon.2013.08.048.

[35] J.M. González-Domínguez, A. Santidrián, A. Criado, C. Hadad, M. Kalbáč, T. Da Ros, Multipurpose nature of rapid covalent functionalization on carbon nanotubes, Chem. Eur. J. 21 (2015) 18631-18641, https://doi.org/10.1002/ chem. 201503085.

[36] J.M. González-Domínguez, A. Ansón-Casaos, L. Grasa, L. Abenia, A. Salvador, E. Colom, J.E. Mesonero, J.E. García-Bordejé, A.M. Benito, W.K. Maser, Unique properties and behavior of nonmercerized type-II cellulose nanocrystals as carbon nanotube biocompatible dispersants, Biomacromolecules 20 (2019) 3147-3160, https://doi.org/10.1021/acs.biomac.9b00722.

[37] C. Ménard-Moyon, C. Fabbro, M. Prato, A. Bianco, One-pot triple functionalization of carbon nanotubes, Chem. Eur. J. 17 (2011) 3222-3227, https://doi.org/ 10.1002/chem.201003050.

[38] J. Mesonero, L. Mahraoui, M. Matosin, A. Rodolosse, M. Rousset, E. Brot-Laroche, Expression of the hexose transporters GLUTI-GLUT5 and SGLTI in clones of Caco-2 cells, Biochem. Soc. Trans. 22 (1994) 681-684, https://doi.org/10.1042/ bst0220681.

[39] F. Zucco, A.-F. Batto, G. Bises, J. Chambaz, A. Chiusolo, R. Consalvo, H. Cross, G. Dal Negro, I. de Angelis, G. Fabre, F. Guillou, S. Hoffman, L. Laplanche, E. Morel, M. Pinçon-Raymond, P. Prieto, L. Turco, G. Ranaldi, M. Rousset, Y. Sambuy, M.L. Scarino, F. Torreilles, A. Stammati, An inter-laboratory study to evaluate the effects of medium composition on the differentiation and barrier function of caco-2 cell lines, Altern. Lab. Anim. 33 (2005) 603-618, https://doi. org/10.1177/026119290503300618. 
[40] J.L. Bahr, J.M. Tour, Highly functionalized carbon nanotubes using in situ generated diazonium compounds, Chem. Mater. 13 (2001) 3823-3824, https:// doi.org/10.1021/cm0109903.

[41] M.S. Strano, C.A. Dyke, M.L. Usrey, P.W. Barone, M.J. Allen, H. Shan, C. Kittrell, R. H. Hauge, J.M. Tour, R.E. Smalley, Electronic structure control of single-walled carbon nanotube functionalization, Science 301 (2003) 1519-1522, https://doi. org/10.1126/science.1087691.

[42] E. Skwarek, Y. Bolbukh, V. Tertykh, W. Janusz, Electrokinetic properties of the pristine and oxidized MWCNT depending on the electrolyte type and concentration, Nanoscale Res. Lett. 11 (2016) 166, https://doi.org/10.1186/ s11671-016-1367-z.

[43] B. White, S. Banerjee, S. O'Brien, N.J. Turro, I.P. Herman, Zeta-potential measurements of surfactant-wrapped individual single-walled carbon nanotubes, J. Phys. Chem. C. 111 (2007) 13684-13690, https://doi.org/10.1021/jp070853e.

[44] L. Grasa, A. Ansón-Casaos, M.T. Martínez, R. Albendea, A. De Martino, S. Gonzalo, M.D. Murillo, Single-walled carbon nanotubes (SWCNTs) enhance KCl-, acetylcholine-, and serotonin-induced contractions and evoke oxidative stress on rabbit ileum, J. Biomed. Nanotechnol. 10 (2014) 529-542, https://doi.org/ 10.1166/jbn.2014.1732.

[45] H. Ali-Boucetta, K.T. Al-Jamal, K.H. Müller, S. Li, A.E. Porter, A. Eddaoudi, M. Prato, A. Bianco, K. Kostarelos, Cellular uptake and cytotoxic impact of chemically functionalized and polymer-coated carbon nanotubes, Small 7 (2011) 3230-3238, https://doi.org/10.1002/smll.201101004.

[46] A. Ansón-Casaos, L. Grasa, D. Pereboom, J.E. Mesonero, A. Casanova, M.D. Murillo, M.T. Martínez, In-vitro toxicity of carbon nanotube/polylysine colloids to colon cancer cells, IET Nanobiotechnology 10 (2016) 374-381, https://doi.org/10.1049/ iet-nbt.2015.0088.

[47] J. Aisner, Overview of the changing paradigm in cancer treatment: oral chemotherapy, Am. J. Heal. Pharm. 64 (2007) 4-7, https://doi.org/10.2146/ ajhp070035.
[48] P. Mathur, S. Rawal, B. Patel, M.M. Patel, Oral delivery of anticancer agents using nanoparticulate drug delivery system, Curr. Drug Metab. 20 (2019) 1132-1140, https://doi.org/10.2174/1389200220666191007154017.

[49] H. Chen, R. Zhao, B. Wang, L. Zheng, H. Ouyang, H. Wang, X. Zhou, D. Zhang, Z. Chai, Y. Zhao, W. Feng, Acute oral administration of single-walled carbon nanotubes increases intestinal permeability and inflammatory responses: association with the changes in gut microbiota in mice, Adv. Healthc. Mater. 7 (2018), 1701313, https://doi.org/10.1002/adhm.201701313.

[50] J. Ciccolini, F. Fina, K. Bezulier, S. Giacometti, M. Roussel, A. Evrard, P. Cuq, S. Romain, P.M. Martin, C. Aubert, Transmission of apoptosis in human colorectal tumor cells exposed to capecitabine, Xeloda, is mediated via Fas, Mol. Cancer Ther. 1 (2002) 923-927.

[51] A. Namvaran, M. Fazeli, S. Farajnia, G. Hamidian, H. Rezazadeh, Apoptosis and caspase 3 pathway role on anti-proliferative effects of scrophulariaoxy sepala methanolic extract on caco-2 cells, Drug Res 67 (2017) 547-552, https://doi.org/ $10.1055 / \mathrm{s}-0043-110483$.

[52] C. Ménard-Moyon, H. Ali-Boucetta, C. Fabbro, O. Chaloin, K. Kostarelos, A. Bianco, Controlled chemical derivatisation of carbon nanotubes with imaging, targeting, and therapeutic capabilities, Chem. Eur. J. 21 (2015) 14886-14892, https://doi. org/10.1002/chem.201501993.

[53] L. Vallan, J. Hernández-Ferrer, L. Grasa, J.M. González-Domínguez, M.T. Martínez, B. Ballesteros, E.P. Urriolabeitia, A. Ansón-Casaos, A.M. Benito, W.K. Maser, Differential properties and effects of fluorescent carbon nanoparticles towards intestinal theranostics, Colloids Surf. B 185 (2020), 110612, https://doi.org/ 10.1016/j.colsurfb.2019.110612.

[54] A. Bianco, K. Kostarelos, C.D. Partidos, M. Prato, Biomedical applications of functionalised carbon nanotubes, Chem. Commun. (2005) 571-577, https://doi. org/10.1039/b410943k. 